\title{
CHK2 Overexpression and Mislocalization within Mitotic Structures Enhances Chromosomal Instability and Hepatocellular Carcinoma Progression
}

\begin{tabular}{|c|c|}
\hline Journal: & Gut \\
\hline Manuscript ID & gutjnl-2016-313114.R2 \\
\hline Article Type: & Original Article \\
\hline Date Submitted by the Author: & $\mathrm{n} / \mathrm{a}$ \\
\hline Complete List of Authors: & $\begin{array}{l}\text { Carloni, Vinicio; Universita degli Studi di Firenze, Department of } \\
\text { Experimental and Clinical Medicine } \\
\text { Lulli, Matteo; University of Florence, Department of Clinical Biomedical } \\
\text { Science, General Pathology Unit } \\
\text { Madiai, Stefania; University of Florence, Department of Experimental and } \\
\text { Clinical Medicine } \\
\text { Mello, Tommaso; University of Florence, Department of Experimental and } \\
\text { Clinical Biochemical Sciences, Gastroenterology Unit } \\
\text { Hall, Andrew; UCL Medical School, Royal Free Campus, Department of } \\
\text { Histopathology } \\
\text { Luong, Tu Vinh; Royal Free Hospital, Department of Cellular Pathology } \\
\text { Pinzani, Massimo; University College London (UCL), Institute for Liver \& } \\
\text { Digestive Health } \\
\text { Rombouts, Krista; University College London, UCL, Institute for Liver \& } \\
\text { Digestive Health, Royal Free } \\
\text { Galli, Andrea; University of Florence, Clinical Pathophysiology, } \\
\text { Gastroenterology }\end{array}$ \\
\hline Keywords: & GENETIC INSTABILITY, HEPATOBILIARY CANCER \\
\hline
\end{tabular}




\title{
CHK2 Overexpression and Mislocalization within Mitotic Structures Enhances Chromosomal Instability and Hepatocellular Carcinoma Progression
}

\author{
Vinicio Carloni ${ }^{1}$,Matteo Lulli ${ }^{2}$, Stefania Madiai ${ }^{1}$, Tommaso Mello ${ }^{3}$, Andrew Hall ${ }^{4}$,Tu Vinh \\ Luong $^{4}$, Massimo Pinzani ${ }^{1,5}$, Krista Rombouts ${ }^{1,5^{*}}$ and Andrea Galli ${ }^{3^{*}}$ \\ ${ }^{1}$ Department of Experimental and Clinical Medicine, ${ }^{2}$ Department of Experimental and \\ Clinical Biomedical Science, General Pathology Unit, ${ }^{3}$ Department of Experimental and \\ Clinical Biochemical Sciences, Gastroenterology Unit, University of Florence, Florence, \\ Italy, ${ }^{4}$ Royal Free Hospital, Department of Cellular Pathology, London, UK, ${ }^{5}$ University of \\ London (UCL)Institute for Liver \&Digestive Health, London, United Kingdom \\ MP and KR have relocated to the University of London, London, UK \\ * These authors contributed equally.
}

Correspondence should be addressed to: Vinicio Carloni MD., PhD, Department of Experimental and Clinical Medicine. University of Florence, Largo Brambilla 3, 50134, Florence, Italy. Phone:+39-55-2758091; Fax:+39-55-7947104; E-mail: vinicio.carloni@unifi.it

Key words: DNA damage response proteins, DNA double-strand breaks, genomic instability, defective chromosome segregation, DNA damage

\begin{abstract}
Abbreviations list: CIN, chromosomal instability; Chk2, checkpoint kinase 2; FHA, forkhead-associated domain; SCD, SQ/TQ cluster domain; hTERT, human telomerase reverse transcriptase; Nek2, NIMA related kinase 2; CENP-H, centromere protein $\mathrm{H}$; $\mathrm{pHH}$, phospho-Histone $\mathrm{H} 3$; Rb, retinoblastoma protein; HA, hemagglutinin; RFP, red fluorescent protein.
\end{abstract}

Count Words: 3938 


\section{Significance of this study}

What is already known on this subject?

- Chromosomal instability (CIN), is a major hallmark of hepatic tumorigenesis and hampers prognosis and therapy. The underlying mechanisms are not well characterized.

- $\quad$ IN is regarded as an ongoing elevated frequency of mitotic errors that leads to numerical and structural chromosome aberrations in a significant proportion of cell divisions leading to intra-tumoral heterogeneity and drug resistance.

- DNA damage caused by genotoxic agents activates DNA damage response checkpoint kinase 2 (Chk2) protein expression

- Mislocalization of proteins, including oncoproteins, tumor suppressors, and other cancer-related proteins, can interfere with normal cellular function and cooperatively drive tumor development

What are the new findings?

- Mitotic errors represented by lagging chromosomes cause DNA damage and overexpress Chk2 throughout hepatic tumorigenesis

- Chk2 and the activation/phosphorylated forms of Chk2 mislocalize within the mitotic structures in the presence of DNA damage contributing to increased numbers of cells with lagging chromosomes. In contrast, knockdown of Chk2 reduces the number of cells with lagging chromosomes and the mitotic index

- The Chk2 forkhead-associated domain (FHA) is responsible for the mislocalization of the protein at the mitotic structures

- Chk2 is expressed at the nuclear level in a subset of human HCC and this aspect correlates with HCC progression. Moreover, CHK2 expression is significantly higher in HCC patients with mutated TP53, a hallmark of genomic instability, than in those with mutated CTNNB1/beta-catenin.

How might it impact on clinical practice in the foreseeable future?

- The study reveals a new mechanistic insight in the co-involvement of Chk2 in CIN i.e. Chk2 mislocalization, hence indicating the possibility to use Chk2 as a new drug target in $\mathrm{HCC}$ 
- The findings of this study suggest Chk2 as a putative biomarker that can be used to detect DNA damage and chromosomal instability in HCC providing a valuable support, not only for prognosis, but also for identifying patients who are likely to have a response to therapy. 


\begin{abstract}
Objective

Chromosomal instability (CIN) is the most common form of genomic instability, that promotes hepatocellular carcinoma (HCC) progression by enhancing tumour heterogeneity, drug resistance and immunity escape. CIN per se is an important factor of DNA damage, sustaining structural chromosome abnormalities but the underlying mechanisms are unknown.
\end{abstract}

\title{
Design
}

DNA-damage response protein Chk2 expression was evaluated in an animal model of diethylnitrosamine-induced HCC characterized by DNA damage and elevated mitotic errors. Chk2 was also determined in two discrete cohorts of human HCC specimens. To assess the functional role of Chk2, gain on- and loss-of-function, mutagenesis, karyotyping, and immunofluorescence/live imaging were performed by using HCT116, Huh7 and human hepatocytes immortalized with hTERT gene (HuS).

\section{Results}

We demonstrate that mitotic errors during HCC tumorigenesis cause lagging chromosomes/DNA damage and activation of Chk2. Overexpression/phosphorylation and mislocalization within the mitotic spindle of Chk2 contributes to induce lagging chromosomes. Lagging chromosomes and mitotic activity are reversed by knockdown of Chk2. Furthermore, up-regulated Chk2 maintains mitotic activity interacting with Aurora B kinase for chromosome condensation and cytokinesis. The forkheadassociated (FHA) domain of Chk2 is required for Chk2 mislocalization to mitotic structures. In addition, retinoblastoma protein phosphorylation contributes to defective mitoses. A cohort and independent validation cohort show a strong cytoplasm-to nuclear Chk2 translocation in a subset of HCC patients.

\section{Conclusions}

The study reveals a new mechanistic insight in the co-involvement of Chk2 in HCC progression. These findings propose Chk2 as a putative biomarker to detect CIN in HCC providing a valuable support for clinical/therapeutical management of patients. 


\section{Introduction}

Hepatocellular carcinoma $(\mathrm{HCC})$ is the primary malignancy of hepatocytes and the most frequent solid tumour of the liver. Half a million cases occur annually and the World Health Organization has recently ranked $\mathrm{HCC}$ as the second most lethal cancer worldwide [1]. Different etiological factors such as hepatitis B and C virus, alcohol and diabetes cause liver injury followed by inflammation, necrosis and hepatocytes proliferation [2]. Continuous cycles of this destructive-regenerative process culminates in liver cirrhosis which is characterized by regenerating nodules that progress to dysplastic nodules and ultimately $\mathrm{HCC}[3,4]$. More than $90 \%$ of $\mathrm{HCC}$, including dysplastic nodules in cirrhotic liver, possess chromosomal aberrations suggesting that chromosomal defects occur at early stages of tumour development and several attempts have been made to connect different chromosomal aberration patterns as specific subsets of HCC, albeit with limited success [5-7]. A common feature of human $\mathrm{HCC}$ and dysplastic nodules is represented by chromosomal instability (CIN). Various types of chromosomal aberrations, including numerical (aneuploidy) and structural (e.g., translocations, deletions), are found in $\mathrm{HCC}$ and are linked to tumorigenesis [8,9]. An important aspect in studying HCC tumorigenesis is to distinguish between the state of the karyotype, and the rate of karyotypic change. In fact, aneuploidy is not synonymous with CIN, because aneuploid tumours are stable with uniform karyotype and phenotype [10-12]. Conversely, there is an increased rate of CIN generating diverse karyotypes within the same tumour. This yields a heterogeneous tumour cell population that has the ability to undergo selective evolution in term of drug resistance and immune system escape mechanisms [13-15]. CIN is regarded as an ongoing elevated frequency of mitotic errors that leads to additional chromosome gains or losses in a significant proportion of cell divisions. Our hypothesis is that CIN per se is also an important mechanism of DNA damage inducing structural chromosome aberrations in HCC. To verify this hypothesis we utilized checkpoint kinase 2 (Chk2) as a central effector of the cell response to DNA damage. In fact, Chk2 has been described to detect a variety of DNA lesions caused by exogenous and endogenous genotoxic agents. Our aim is to investigate the expression of this kinase in hepatic carcinogenesis and its correlation with the genesis of structural chromosomal 
aberrations. Here, we provide evidence that defective chromosome segregation i.e. lagging chromosomes causes DNA damage and determines an overexpression/phosphorylation of Chk2. Furthermore, the mislocalization of Chk2 with components of the mitotic spindle contributes to chromosome missegregations. These data are corroborated with the observation of an increased Chk2 and nuclear translocation in two independent cohorts of human HCC specimens. 


\section{Materials and Methods \\ Please refer to the Supplementary Materials and Methods for more detailed descriptions.}

\section{Tissue Samples and Immunohistochemistry Assays.}

Florence cohort, University Hospital of Florence; formalin-fixed, paraffin-embedded tissue were obtained from an archive of liver biopsies of patients diagnosed with HCC. H\&E-stained tissue sections of 49 patients were reviewed, and areas of cirrhotic and $\mathrm{HCC}$ were identified. Chk2 antigen retrieval was performed by heating in citrate buffer $\mathrm{pH} 6.0$ at $98^{\circ} \mathrm{C}$ for 30 minutes. Non-specific signal was eliminated by peroxidase block for 10 minutes at RT. Primary antibody was incubated at room temperature overnight in a humidified chamber followed by HRP-conjugated secondary antibody incubation for 30 minutes at room temperature. Antibody binding was revealed by DAB and reaction was stopped by immersion of tissue sections in distilled water once brown colour appeared. Tissue sections were counterstained by hematoxylin, dehydrated in graded ethanols and mounted. All reagents for immunohistochemistry were from Dako. Appropriate positive and negative controls were included for each run of immunohistochemistry. Chk2 expression was determined according to $\mathrm{H}$-score method. For $\mathrm{H}$-score assessment, 10 fields were chosen at random at $\times 400$ magnification and the staining intensity in the malignant cell nuclei was scored as $0,1,2$, and 3 corresponding to the presence of negative, weak, intermediate, and strong brown staining, respectively. The total number of cells in each field and the number of cells stained at each intensity were counted. The average percentage positive was calculated, and a $\mathrm{H}$-score between 0 and 300 was obtained where 300 was equal to $100 \%$ of tumor cells stained brown strongly. The positive cut off was considered $\geq 100$ (supplemental Figure S1). Chk2 expression in biopsy sections was scored by a pathologist and two researchers independently.

London cohort, Royal Free Hospital; formalin-fixed, paraffin-embedded surgical specimens from consecutive patients diagnosed with $\mathrm{HCC}$ were retrieved from Royal Free Hospital archives. All samples were stained with H\&E and the sections were 
reviewed and suitable areas of cirrhosis and HCC were identified in 20 samples. Antigen retrieval was performed by microwaving $(720 \mathrm{~W})$ in $\mathrm{pH} 6.0$ sodium citrate buffer for 30 mins. Endogenous peroxidase and non-specific binding of primary antibody was blocked. The primary Chk2 antibody was incubated at overnight at $5^{\circ} \mathrm{C}$. Primary antibody detection was carried out using the Novolink polymer detection system (Novocastra).Tissue sections were counterstained with Mayer's hematoxylin.

Experimental hepatocarcinogenesis.Twenty-five female Wistar rats (210-225 g) were given diethylnitrosamine $50 \mathrm{mg} / \mathrm{L}$ ad libitum in their drinking water for a period of 10 weeks as previously described [16]. Another group of five animals were maintained as controls. All animals were administered a standard laboratory diet and were weighed each week. Five animals for each time point were sacrificed on week 4, 6, 8, 10 and, 14.Tumours and liver tissues were removed and fixed in 10\% neutral buffered formalin. Liver sections $(5 \mu \mathrm{m}) \quad$ were cut from paraffin-embedded blocks and stained Chk2 (Abcam, \#ab47433), phospho-Histone H2A.X (Cell Signaling, \#9718) and hematoxylin and eosin (H\&E) for histological examination and expression were determined according to $\mathrm{H}$-score method.

\section{CHK2 Expression in HCC samples from Cancer Genome Atlas (TCGA) database.}

The TCGA-LIHC cohort, containing 377 cases, was used to validate CHK2 expression levels in distinct subtypes of HCC. The TCGA database (https://gdc-portal.nci.nih.gov) was accessed on November 16, 2016 through the cGbioportal website (http://www.cbioportal.org/) to retrieve patients with either TP53 or CTNNB1 mutation. The TCGA_participant_barcode was used to download CHK2 expression from RNA-seq data using the firebrowse portal (http://firebrowse.org). Patients with co-occurrent mutations of TP53 and CTNNB1 were excluded from the analysis. CHK2 expression levels were retrieved for 101 patients with TP53 mutation and 85 patients with CTNNB1 mutation. Statistical analysis was performed using the Mann-Whitney test with GraphPad 6 software. 


\section{Results}

\section{Chk2 is overexpressed in a subset of human HCC}

To test our hypothesis that hepatic tumorigenesis per se could be a cause of DNA damage and structural chromosome aberrations, an animal model of diethylnitrosamineinduced HCC was employed which induced macroscopical visible tumours after 14 weeks (Figure 1A). This model has previously been established to induce elevated mitotic errors, DNA damage and chromosome aberrations [17]. Using this model we can compare the expression levels of DNA damage response proteins induced by the genotoxic activity of diethylnitrosamine with a later phase represented exclusively by tumorigenesis. Therefore, Chk2, a DNA damage-response checkpoint kinase, and phospho-Histone H2A.X (Ser139) a component of chromatin which becomes phosphorylated following DNA damage (e.g. DNA double-stranded breaks) were investigated [18]. As shown in Figure 1B several nuclei of cancer cells exhibited an increased expression of Chk2 and phospho-Histone H2A.X revealing the presence of DNA damage. A timeline analysis of liver sections demonstrated a two-fold increase in Chk2 basal levels at 4 weeks followed by decreased values after 8 weeks (Figure 1C). In contrast, by 14 weeks tumours were macroscopically visible and histologically evident in $80 \%$ of animals. Immunostaining of HCC sections showed a significant increased expression of Chk2 and phospho-Histone H2A.X (Figure 1C and 1D) when compared with liver sections without evidence of tumours (8 weeks). Taken together, these data showed that the increased expression of Chk2 reflected a response triggered by DNA damage during tumorigenesis.

To extend these findings to humans, the expression of Chk2 in patients with cirrhoticrelated HCC and normal liver was examined in liver biopsies from hospital archives (Florence cohort, supplemental Table 1). A nuclear staining of Chk2 was detectable in distinctive histotypes of HCC (supplemental Figure S2 and S3). More specifically, the expression level of Chk2 in different grades of HCC was evaluated. Remarkably, within histological grade II and III, significant quantitative differences between positive and negative Chk2 patients were found $(p<0.001)$. Overall, these data demonstrated that 
Chk2 nuclear positivity was found in $63.2 \%$ (31 of 49) of HCCs analyzed (supplemental Table 2 ). A separate validation cohort of patients was examined at Royal Free Hospital, London (supplemental Table 3). Normal liver tissue showed a moderate and diffuse cytoplasmic staining, whereas Grade 1 specimens were characterized with weak focal cytoplasmic Chk2 staining. In Grade 2, a Chk2 perinuclear and nuclear accumulation was observed. Specimens with Grade 3 showed exclusively a clear and strong nuclear staining, without cytoplasmic and perinuclear Chk2 staining (Figure 2a and supplemental Table 4). Furthermore, the expression levels of CHK2 were analyzed in a public available cohort of HCC from the TCGA database. We found a highly-significant increase of CHK2 expression in HCC with mutated TP53, a hallmark of genomic instability, with respect to those with CTNNB1/beta-catenin mutation (Figure 2b).

\section{Defective chromosome segregations cause DNA damage}

The previous observations prompted us to investigate the functional role of Chk2. For this reason, three different cell lines were employed. The colon carcinoma cell line HCT116 was used i.e. a near-diploid cell line that faithfully segregates chromosomes to maintain a stable karyotype and is commonly employed in CIN studies [19,20]. Secondly, the HCC cell line Huh7 was used which is a stable hyperdiploid karyotype with altered chromosomal structures and finally HuS cells were used marked with an unstable karyotype [21]. HuS cells are human hepatocytes immortalized with human telomerase reverse transcriptase (hTERT), a gene described commonly activated in human HCC $[8,9]$. HuS cells just established in culture ( 0 generation) and hereafter referred to as HuSOgen showed a karyotype with a distribution of 46 chromosomes (Figure $3 \mathrm{~A}$ ) and expressed low levels of Chk2 protein in comparison to HCT116 and Huh7 (Figure 3B). The HuSOgen cells were maintained in culture and after 30 consecutive generations (HuS30gen) were re-examined to verify the karyotype status. The cells showed a distribution near-triploid, with many structural chromosomal defects (Figure $3 \mathrm{C}$ and supplemental Figure S4A) and importantly this aspect was associated with increased expression of Chk2 protein, (Figure 3B). Likewise with the murine model, we evaluated the expression levels of phospho-Histone H2A.X (Ser139) in all 
the cell lines studied. As shown in Figure 3D, both HuSOgen and HuS30gen cells exhibited high levels of phospho-Histone H2A.X, although with different levels of expression. These findings indicate the presence of DNA double-strand breaks, a hallmark of DNA damage, in HuS cells.

Overexpressed Chk2 mislocalizes with mitotic structures in HuS30gen cells but not in HCT116 and Huh7.

The finding of overexpressed Chk2 in both karyotyped stable HCT116 and Huh7 and in unstable karyotyped HuS30gen did not demonstrate a specific role for Chk2 in defective chromosome segregation. Therefore, we analyzed the cell lines HCT116, Huh7, HuSOgen and HuS30gen by immunofluorescence to examine a possible altered localization of Chk2 with the mitotic structures. Chk2 showed a localization to the spindle poles of metaphase mitotic structures in HCT116, Huh7, and HuS0gen (Figure 4A). Very importantly, HuS30gen cells showed a robust localization of Chk2 with all metaphase mitotic structures indicating a clear mislocalization of Chk2 (Figure 4A and supplemental Figure S4B). These results suggest that in HuSOgen cells, expressing low levels of DNA damage, Chk2 weakly co-localized with the mitotic spindle components, whereas Chk2 is increased and strongly associated with all mitotic structures in HuS30gen cells that are expressing high levels of DNA damage. To further address these findings we stably transfected HuSOgen with HA-tagged Chk2, HuSOgen overexpressing Chk2 exhibited Chk2 localization with mitotic structures as shown in Figure 4B. Next, we measured the number of lagging chromosomes (Figure 4C) in HuSOgen, HuS30gen, and in HuSOgen stably transfected with HA-tagged Chk2 to mimic overexpression of Chk2. As shown in Figure 4D, overexpression of Chk2 significantly correlated with high number of mitoses with lagging chromosomes in HuS30gen, and in HuSOgen overexpressing HA-tagged Chk2. These data clearly indicate that indeed overexpression and mislocalization of Chk2 itself contributes to defective chromosome segregations. Furthermore, to assess that the increased expression of Chk2 was specifically related to those mitoses with defective chromosome segregations and not a random deregulation of proteins, the expression of Nek2 (NIMA related kinase 2) protein was evaluated. Nek2 is a protein located at the 
centrosome/spindle poles and responsible for chromosomal defective segregations [22]. HCT116 and Huh7 cells showed a strong Nek2 expression whereas both HuSOgen and HuS30gen cells expressed low levels of Nek2 (supplemental Figure S5) suggesting that the previously observed overexpression of Chk2 in Hus30gen was a specific event that coincided with structural chromosomal defects and DNA damage.

Chk2 overexpression is correlated with increased chromosomal missegregations The findings that HuS30gen express high levels of Chk2 protein correlating with DNA damage (Figure 3B and $3 \mathrm{D}$ ), prompted us to investigate the effects of knocking down expression of CHK2 in HuS30gen cells by retroviral-mediated short hairpin RNA (shRNA) transduction. A pool of four shRNA targeting Chk2 resulted in effective decrease of the protein (Figure $5 \mathrm{~A}$ ). It is important to note that silencing $\mathrm{CHK} 2$ coincided with a significant reduction of the number of HuS30gen cells with lagging chromosomes and HuS30gen mitotic index. To address any off target effects of the hairpins, we rescued the mitotic index and the number of cell with lagging chromosomes of the knockdown HuS30gen by expressing a myc-tagged version of Chk2 that circumvents the shRNA directed against human Chk2 (Figure 5B and $5 \mathrm{C}$ ). These data demonstrate that Chk2 overexpression is correlated with increased chromosomal missegregation and mitotic index.

\section{Defective mitoses-related DNA damage promotes Chk2 phosphorylation and mislocalization within mitotic structures.}

Human CHK2, is a 543 amino acid protein that consists of a N-terminal SQ/TQ cluster domain ( $\mathrm{SCD}$ ), a central forkhead-associated (FHA) domain, and a C-terminal serine/threonine kinase domain [23]. The SCD consists of multiple SQ/TQ (Ser-Gln/ThrGIn) motifs with Thr68 being the primary site to be phosphorylated in response to DNA damage followed by phosphorylation of Thr387 in the activation loop of the kinase domain $[24,25]$. Therefore, the protein expression of phospho-Thr68-Chk2 and phospho-Thr387-Chk2 in HuS30gen and HuS0gen stably transfected with HA-tagged 
Chk2 were assessed. As shown in Figure 5D phospho-Thr68-Chk2 and phosphoThr387-Chk2 were highly expressed in HuS30gen and in HuSOgen stably transfected with HA-Chk2, but not in HuS0gen as expected. Furthermore, phospho-Thr68-Chk2 revealed a robust localization with components of the mitotic spindle as previously shown for Chk2. In particular, a prominent association of phospho-Thr68-Chk2 was observed with mitotic structures such as the spindle poles, kinetochores and midbody in Hus30gen cells, (Figure 5E, upper pane/s). In contrast, Huh7 cells expressing low levels of DNA damage ( see Figure 3D) did not show co-localization of phosphoThr68-Chk2 with mitotic structures, (Figure 5E, lower panels). Taken together, these results reveal an overexpression/mislocalization of phosphorylated Chk2, which is correlated with its activation status, and manifestations of lagging chromosomesinduced DNA damage.

\section{Up-regulated Chk2 cooperates with Aurora B for chromosome condensation and cytokinesis.}

As previously shown the typical localization of activated Chk2 in HuS30gen cells was observed in kinetochores and specifically the midbody, suggesting a possible interaction with Aurora B kinase. This protein is known to be involved in chromosome condensation, the assembly of the spindle midzone in anaphase and the midbody during telophase. Furthermore, Aurora B kinase has been identified as an effective predictor of aggressive HCC recurrence, in relation to genomic instability [26]. Therefore, HuSogen cells were transfected with HA-tagged Chk2 and immunofluorescence analyses revealed a co-localization of Chk2 with Aurora B throughout all mitotic phases, and this co-localization was also present in cells marked with lagging chromosomes (Figure $6 \mathrm{~A}$ and $6 \mathrm{~B}$ ). Aurora $\mathrm{B}$ is responsible for the phosphorylation of histone $\mathrm{H} 3(\mathrm{pHH} 3)$, and $\mathrm{pHH} 3$ serine residue (Ser-10) is considered to be a crucial event for the onset of mitosis. The utility of $\mathrm{pHH} 3$ to identify mitoses has been demonstrated in several cancer studies [27-29]. As, the co-localization of Aurora B with Chk2 was demonstrated we further explored the co-localization of overexpressed HA-Chk2 with pHH3. As shown in Figure 6C, Chk2 exhibited a striking co-localization 
with $\mathrm{pHH} 3$. Overall, the data so far demonstrate that in unstable HuS cells during mitosis, lagging chromosomes cause DNA damage, overexpression of Chk2 which localizes to all mitotic structures. This overexpressed and mislocalized Chk2 protein contributes to a constant mitotic activity and more important Aurora B kinase and $\mathrm{pHH} 3$ interact with Chk2. This favours a possible scenario where an overexpressed and mislocated Chk2 overrules the signalling specificity at distinct mitotic checkpoints inducing itself chromosomal aberrations, hence CIN.

\section{The forkhead associated domain (FHA) determines Chk2 mislocalization to mitotic structures and is essential for DNA damage response}

To understand how Chk2 is recruited to the mitotic structures in such a specific manner, we investigated Chk2 specific domain(s) that are required for Chk2 localization. A series of RFP-tagged Chk2 variants were created, each variant carrying a point mutation or a deletion, and were expressed and localized individually in HuS30gen. The RFP-Chk2D347A, a kinase-inactive mutant, localized similarly as shown for RFP-Chk2 (Figure 7A). This indicated that the kinase activity is dispensable for Chk2 mislocalization (Figure 7B). The FHA domain of Chk2 has been proposed to mediate localization of Chk2 to mitotic structures for the reason that FHA domains function as phospho-threonine-binding protein motifs. The deletion of the Chk2 FHA domain disrupted the localization of the protein to mitotic structures such as the spindle poles and kinetochores indicating that the deletion of the FHA is responsible for the observed mislocalization (Figure 7C). Indeed, the entire FHA domain RFP-tagged localized to mitotic structures similar to RFP-Chk2 (Figure 7D). Along these lines, we can conclude that the FHA domain is required for proper Chk2 localization to mitotic structures. Furthermore, we analyzed the effects of the several Chk2 mutants on lagging chromosomes. Interestingly, transfection of HuSOgen with Chk2 FHA domain significantly reduced the number of lagging chromosomes (Figure 7E). This result suggests a dominant negative effect of Chk2 FHA domain towards endogenous Chk2.

Rb phosphorylation contributes to defective mitoses in HuS cells. 
An important point raised by defective mitoses-related DNA damage as observed in this study is why the cells do not sense signals deployed to curb cell proliferation, as normally the growth of cultured diploid cells is halted by chromosome missegregation. In HCC, p53 deficiency has been described to participate in the development of CIN, however the relevance of $\mathrm{p} 53$ to $\mathrm{HCC}$ initiation, progression, or both, remains an area of active investigation [2]. Therefore, the basal level of p53 in HCT116, Huh7, HuS30gen and HuSOgen was investigated. As shown in Figure 8A, p53 was expressed in Huh7 and at low level in HuS30gen cells. HuSOgen cells did not express p53 in basal conditions, however treatment of the cells with 5-fluorouracil, a DNA-damaging agent, triggered a strong p53 protein expression. Based on these findings we assume that p53 expression may be a late event in HuS cells, as a result of several defective mitoses [19]. Furthermore, mutated p53 was not present in HCT116 and HuS30gen cells in contrast to Huh7 cells that we used as a positive control (Figure 8B) [30].

The possible involvement of the retinoblastoma protein $\mathrm{Rb}$ was evaluated since several studies have described $\mathrm{Rb}$ as an important regulator of cell division [31]. Specifically, $\mathrm{Rb}$ phosphorylation determines $\mathrm{Rb}$ inactivation and leads to defective mitoses [31]. Therefore, we evaluated the protein expression levels of phospho-Rb (Ser807/811) in HCT116, HuSOgen cells and HuS30gen. As shown in Figure 8C HuS30gen cells express higher levels of phospho-Rb (Ser807/811) in comparison to HuSogen cells. Moreover, HCT116 (Figure 8D), HuS30gen (Figure 8E) and HuSOgen (Figure 8F) exhibited a localization of phospho-Rb (Ser807/811) at the chromosomal level. Taken together, these findings suggest that in HuS30gen $\mathrm{Rb}$ phosphorylation is required to sustain mitotic activity which leads to mitotic defects and DNA damage. 


\section{Discussion}

The neoplastic evolution of HCC proceeds through a multi-step histological process that is less well defined than that of other cancer types. Hyperplastic nodules of regenerating hepatocytes have normal karyotype and represent a potential first step towards HCC. These lesions can progress to dysplastic nodules, which have abnormal chromosome features including numerical and structural alterations. These dysplastic nodules can evolve to HCC marked with recurrent regions of copy number change and allelic imbalances [3, 4]. Loss of certain tumour suppressors or gain of specific oncogenes promote cell division as shown in many previous studies [25, 32]. We demonstrate that this coincides with defective mitoses which can cause DNA damage. This scenario is conceivable considering that massive deregulation of mitosis is incompatible with cell survival and can even be tumour suppressive [33]. Therefore, subtle rather than massive mitotic defects are expected to underlie CIN in HCC, allowing a tolerable level of chromosome defects. To support this view we have provided evidence in this study that hepatocarcinogenesis with lagging chromosomes elicits the expression of DNA damage response protein Chk2. Thus, the overexpression of Chk2 and its mislocalization within structures of the mitotic spindle contribute to sustain cell division and chromosomes missegregation [34-36]. This forms a self-perpetuating mechanism causing DNA damage (Figure 9).

We have also found that functional $\mathrm{Rb}$ inactivation is necessary but not sufficient to trigger chromosome missegregation. Indeed, phosphorylation of $\mathrm{Rb}$ deregulates transcription factor E2F, and well-characterized targets of E2F include multiple genes whose products are required to maintain cell division [37-39]. The integrity of chromosomal DNA is under constant surveillance throughout the cell division. Surprisingly, the fact that a deregulated functional Chk2 itself might cause per se CIN through abnormal spindle localization is intriguing, as the role of Chk2 in the maintenance of genetic stability has mainly been ascribed to its function in DNA damage checkpoint signalling.

When DNA damage occurs, Chk2 is first activated in the nucleus during interphase, spreads to the cytoplasm and localizes to the various mitotic structures because of the 
phosphopeptide-binding ability of the FHA domain [40]. During DNA damage, chromosomes accumulate a number of phosphoproteins around DNA damage sites [41]. Chk2 may bind to specific phosphoproteins through the FHA domain forming numerous chromosomal foci and trap Chk2 to the structures. When this FHA domain is deleted, the molecule is excluded from the mitotic chromosomes and therefore does not mislocalize to kinetochores, spindle poles and midbody as was demonstrated in Figure 7. Moreover, Chk2 localization to the kinetochores and midbody region suggests that Chk2 may directly affect spindle assembly function, similar to Aurora B kinase [42], but only when DNA damage exists. The data obtained in this study fit a model whereby abnormal expression of Chk2 and Aurora B kinase disrupts the sensitive balance of mitotic proteins, which in turn undermines faithful chromosome segregation [34]. If the relative expression levels of the proteins involved in the quality control of the machinery which ensures chromosome segregation fidelity is disrupted by mitotic errors and DNA damage, the resulting imbalance could further compromise chromosome segregation accuracy.

Since Chk2 overexpression can induce oligomerization and cis- and trans phosphorylation of regulatory residues $[43,44]$, one possibility is that local enrichment of Chk2 at kinetochores and midbody regions facilitates Chk2 oligomerization and activation. This mechanism may alter regulation of Chk2-dependent processes such as centrosome function, cell cycle progression, and chromosome segregation. The discovery of a role for Chk2 in HCC, its association with DNA damage and CIN may have potential diagnostic and prognostic significance. Importantly, grading heterogeneity inside a tumour is frequently observed and may significantly limit diagnosis and choice of treatment. This study suggests that stratification of HCC patients according to CIN status and/or DNA damage may be useful to minimize the confounding effects of tumor CIN status in clinical trials. Along these lines, we propose that the diagnostic and prognostic value of Chk2 should be further verified in prospective clinical trials. 
Competing interests: The authors have declared that no conflict of interest exists.

Contributors: V.C. study concept and experimental design, cloning experiments, analysis and interpretation of data, drafting of the manuscript, study supervision; M.L. immunoblotting and karyotype experiments; S.M. immunohistochemistry and animal experiments; T.M. immunofluorescence, live-cell, bioinformatics analysis; A.H. immunohistochemistry analysis; TV.L. human tissue evaluation; M.P. critical review of the manuscript; K.R. experimental design, data analysis, critical review of the manuscript; A.G conceptual and intellectual input, critical review of the manuscript. All authors read and approved the final manuscript.

Funding: This study was in part supported by the University of Florence and Regione Toscana "Ricerca regionale in materia di salute D.D. n. 3242 ", and by Ministero dell'Istruzione, dell'Università FIRB RBAP10MY35_002 and FiorGen Foundation. 


\section{References}

[1] McGlynn KA, Petrick JL, London WT. Global epidemiology of hepatocellular carcinoma: an emphasis on demo graphic and regional variability. Clin Liver Dis 2015;19:223-238

[2] Farazi PA, DePinho RA. Hepatocellular carcinoma pathogenesis: from genes to environment. Nat Rev Cancer. 2006;6:674-87.

[3] Carloni V, Luong T V, Rombouts K. Hepatic stellate cells and extracellular matrix in hepatocellular carcinoma: more complicated than ever. Liver Int. 2014;34:834-843.

[4] Mazzocca A, Liotta F, Carloni V. Tetraspanin CD81-regulated cell motility plays a critical role in intrahepatic metastasis of hepatocellular carcinoma. Gastroenterology 2008; 135:244-256

[5] Sakakura C, Hagiwara A, Taniguchi $\mathrm{H}$, et al. Chromosomal aberrations in human hepatocellular carcinomas associated with hepatitis $C$ virus infection detected by comparative genomic hybridization. Br. J. Cancer 1999;80: 2034-2039.

[6] Wong N, Lai P, Pang E, et al. A comprehensive karyotypic study on human hepatocellular carcinoma by spectral karyotyping. Hepatology 2000;32: 1060-1068.

[7] Kusano N, Shiraishi K, Kubo K, et al. Genetic aberrations detected by comparative genomic hybridization in hepatocellular carcinomas: their relationship to clinic pathological features. Hepatology.1999;29:1858-62

[8] Shimojima $M$, Komine $F$, Hisatomi $H$, et al. Detection of telomerase activity, telomerase RNA component, and telomerase reverse transcriptase in human hepatocellular carcinoma. Hepatol. Res. 2004; 29: 31-38.

[9] Youssef N, Paradis V, Ferlicot S, et al. In situ detection of telomerase enzymatic activity in human hepatocellular carcinogenesis. J. Pathol. 2001;194: 459-465.

[10] Kawai, H. Suda T, Aoyagi Y, et al. Quantitative evaluation of genomic instability as a possible predictor for development of hepatocellular carcinoma: comparison of loss of heterozygosity and replication error. Hepatology 2000; 31: 1246-1250.

[11] Guan XY, Fang Y, Sham JS, et al. Recurrent chromosome alterations in hepatocellular carcinoma detected by comparative genomic hybridization. Genes Chromosomes Cancer 2000; 29:110-116. 
[12] Wilkens L, Flemming $P$, Gebel $M$, et al. Induction of aneuploidy by increasing chromosomal instability during dedifferentiation of hepatocellular carcinoma. Proc. Natl Acad. Sci. USA. 2004;101: 1309-1314

[13] Almendro V, Marusyk A, Polyak K. Cellular heterogeneity and molecular evolution in cancer. Annu Rev Pathol. 2013;8:277-302.

[14] Bakhoum F S, Compton A D. Chromosomal instability and cancer: a complex relationship with therapeutic potential. J Clin Invest. 2012;122:1138-1143

[15] Carloni V, Mazzocca A, Mello T, et al. Cell fusion promotes chemoresistance in metastatic colon carcinoma. Oncogene. 2013; 32: 2649-266

[16] Carloni V, Vizzutti F, Pantaleo P. Farnesyltransferase inhibitor ABT-100 is a potent liver cancer chemopreventive agent. Clin Cancer Research. 2005; 11: 4266-4274.

[17] Basak R, Saha BK,Chatterjee M. Inhibition of diethylnitrosamine-induced rat liver chromosomal aberrations and DNA-strand breaks by synergistic supplementation of vanadium and 1a,25-dihydroxyvitamin D3. Biochim Biophys Acta. 2000;1502:273-282 [18] Rogakou EP, Pilch DR, Orr AH, et al. DNA double-stranded breaks induce histone H2AX phosphorylation on serine 139. J Biol Chem. 1998;273:5858-68

[19] Thompson SL, Compton DA. Proliferation of aneuploid human cells is limited by a p53-dependent mechanism. J Cell Biol. 2010; 188:369-381

[20] Stolz A, Ertych N, Kienitz A, et al. The CHK2- BRCA1 tumour suppressor pathway ensures chromosomal stability in human somatic cells. Nat Cell Biol. 2010 ;12:492-499.

[21] Durantel D, Zoulim F. Going towards more relevant cell culture models to study the in vitro replication of serum-derived hepatitis $C$ virus and virus/host cell interactions $\mathrm{J}$ Hepatol 2007; 46:1-5

[22 ] Hayward DG, Fry AM. Nek2 kinase in chromosome instability and cancer. Cancer Lett. 2006;237:155-166.

[23] CaiZ, Chehab NH, Pavletich NP. Structure and activation mechanism of the CHK2 DNA damage checkpoint kinase. Mol Cell. 2009;35:818-29

[24] Ward IM, WuX, Chen J. Threonine 68 of Chk2 is phosphorylated at sites of DNA strand breaks. J Biol Chem. 2001;276:47755-8 
[25] Janssen A, van der Burg M, Szuhai K, et al. Chromosome segregation errors as a cause of DNA damage and structural chromosome aberrations. Science. 2011;333:1895-1898

[26] Adams RR, Carmena M, Earnshaw WC. Chromosomal passengers and the (aurora) ABCs of mitosis. Trends Cell Biol 2001; 11: 49-54.

[27] Hayashi-TakanakaY, Yamagata K, Nozaki N, et al. Visualizing histone modifications in living cells: spatio-temporal dynamics of H3 phosphorylation during interphase. J Cell Biol. 2009; 187:781-790

[28] Crosio C, Fimia GM, Loury R, et al. Mitotic phosphorylation of histone H3: spatiotemporal regulation by mammalian Aurora kinases Mol Cell Biol. 2002;22:874-85

[29] Ladstein RG, Bachmann IM, Straume O, et al. Prognostic importance of the mitotic marker phospho-histone $\mathrm{H} 3$ in cutaneous nodular melanoma. J Invest Dermatol. 2012;132:1247-1252.

[30] Mazzocca A, Giusti S, Hamilton AD, et al. Growth inhibition by the farnesyltransferase inhibitor $\mathrm{FTI}-277$ involves $\mathrm{Bcl}-2$ expression and defective association with Raf-1 in liver cancer cell lines. Mol Pharmacol. 2003;63:159-166

[31] Manning AL, Dyson NJ. pRB, a tumor suppressor with a stabilizing presence. Trends Cell Biol. 2011;21:433-41

[32] Baker DJ, Jin F, Jeganathan KB, et al. Whole chromosome instability caused by Bub1 insufficiency drives tumorigenesis through tumor suppressor gene loss of heterozygosity. Cancer Cell. 2009;16:475-486.

[33] Weaver BA, Silk AD, Montagna C, et al. Aneuploidy acts both oncogenically and as a tumor suppressor. Cancer Cell. 2007; 11:25-36.

[34] Lin ZZ, Jeng YM, Hu FC, et al. Significance of Aurora B overexpression in hepatocellular carcinoma. Aurora B overexpression in HCC. BMC Cancer. 2010;10:461

[35] Tanaka S, Arii S, Yasen M, et al. Aurora kinase B is a predictive factor for the aggressive recurrence of hepatocellular carcinoma after curative hepatectomy. $\mathrm{Br} \mathrm{J}$ Surg. 2008; 95: 611-619

[36] Lu G, ShanT, He S, et al. Overexpression of CENP-H as a novel prognostic biomarker for human hepatocellular carcinoma progression and patient survival. Oncol Rep. 2013;30:2238-44. 
[37] Burkhart DL, Sage J. Cellular mechanisms of tumour suppression by the retinoblastoma gene. Nat Rev Cancer.2008;8:671-82

[38] Genovese C,Trani D, Caputi M, et al. Cell cycle control and beyond: emerging roles for the retinoblastoma gene family. Oncogene. 2006;25:5201-9.

[39] van Harn $T$, Foijer $F$, van Vugt $M$, et al. Loss of Rb proteins causes genomic instability in the absence of mitogenic signaling. Genes Dev. 2010;24:1377-88

[40] Takeda S, Collins ER, Kurahashi K. The FHA domain determines Drosophila Chk2/Mnk localization to key mitotic structures and is essential for early embryonic DNA damage responses. Mol Biol Cell. 2015 ;26:1811-1828.

[41] Polo SE, Jackson SP. Dynamics of DNA damage response proteins at DNA breaks: a focus on protein modifications. Genes Dev 2011;25: 409-433.

[42] Fuller BG, Lampson MA, Foley EA, et al. Midzone activation of aurora $B$ in anaphase produces an intracellular phosphorylation gradient. Nature. 2008; 453:11321136

[43] Schwarz JK, Lovly CM, Piwnica-Worms H. Regulation of the Chk2 protein kinase by oligomerization-mediated cis- and trans-phosphorylation. Mol. Cancer Res. 2003;1: 598-609.

[44] Li J, Williams BL, Haire LF, et al. Structural and functional versatility of the FHA domain in DNA-damage signaling by the tumor suppressor kinase Chk2. Mol Cell. 2002;9:1045-54. 
Figure legends

Figure 1. Immunohistochemical detection of Chk2 and phospho-Histone H2A.X(Ser139) in diethylnitrosamine-induced HCC. A) Representative image of livers of female Wistar rats used as control (left panel) and rats HCC (right panel) which were given diethylnitrosamine $50 \mathrm{mg} / \mathrm{L}$ ad libitum in their drinking water for a period of 10 weeks. Scale bar, $10 \mathrm{~mm}$. B) Liver sections (HCC 14 weeks) were analyzed for Chk2 and phospho-Histone H2A.X(Ser139) expression (see nuclear staining in the right panels, Scale bar $200 \mu \mathrm{m}$.). C) and D) Five animals for each time point were sacrificed on week 4, 6, 8, 10, 14. Chk2 and phospho-Histone H2A.X(Ser139) expression were determined according to $\mathrm{H}$-score method. The average positive percentage was calculated, and a H-score between 0 and 300 was obtained where 300 was equal to $100 \%$ of tumour cells stained brown strongly. The positive cut off was considered $\geq 100$. The levels of Chk2 and phospho-Histone H2A.X(Ser139) expression are indicated as fold increase of cut-off values. ${ }^{*} P<0.001$ vs 8 weeks. Chk2 expression was evaluated in the nucleus of the HCC tissue as indicated by the brown staining.

Figure 2a. Detection of Chk2 protein in human HCC by immunohistochemistry. Paraffin-embedded tissue sections were stained with a specific antibody for Chk2 and hematoxylin and eosin. A) Normal liver with a moderate, diffuse cytoplasmic Chk2 staining. B) Chk2 immunoreactivity in cancer tissue from a tumour classified Grade 1 characterized by a weak focal cytoplasmic Chk2 staining. C) and D) Chk2 immunoreactivity in cancer tissue from a tumour classified Grade 2 with a strong Chk2 perinuclear and moderate nuclear accumulation. E) Chk2 immunoreactivity in cancer tissue from a tumour classified Grade 3 with a clear and strong nuclear staining, without cytoplasmic and perinuclear Chk2 staining. Scale bar $100 \mu \mathrm{m}$.

\section{Figure 2b. CHK2 Expression in HCC subtypes from TCGA database.}

CHK2 expression from RNA-seq data was retrieved for 101 patients with TP53 mutations and 85 patients with CTNNB1/beta-catenin mutations, using the LIHC cohort of 377 cases (accessed on November 16, 2016). Patients with both TP53 and CTNNB1 
mutations were excluded from the analysis. Expression levels are expressed as log2exp (absolute levels) or z-score (expression levels in HCC samples normalized to the expression levels in the reference population).Graph shows mean \pm SEM, ${ }^{*} p<0.0001$

Figure 3. Defective chromosome segregation causes Chk2 overexpression in hTERT immortalized human hepatocytes. A) Examples of chromosome numbers from metaphase spreads of HCT116, Huh7 and HuS0gen cells (upper panels). In the bottom panels individual chromosome numbers percentage from metaphase spreads of HCT116, Huh7 and HuSOgen cells are represented $(n=20)$. B) Representative immunoblot out of three independent experiments demonstrating the protein levels of Chk2 in indefinite cultured HCT116 and Huh7 cells, in HuSOgen cells and HuSOgen cells after 30 generations in culture (HuS30gen). C) Example of chromosomes from metaphase spreads of HuS30gen exhibiting numerical and structural (red arrow) alterations; the histogram shows individual chromosome numbers percentage in HuS30gen ( $n=20)$. D) Representative immunoblot out of three independent experiments demonstrating the protein levels of phospho-Histone H2A.X (Ser139), a hallmark of DNA damage, in indefinite cultured HCT116, Huh7, HuSOgen and HuS30gen cells.

Figure 4. Chk2 overexpression is linked to lagging chromosomes. A) Chk2 colocalizes with chromosomal structures only in HuS30gen cells. Asynchronously growing HCT116, Huh7, HuS0gen and HuS30gen cells were synchronized in metaphase by releasing cells from thymidine treatment into MG132. Metaphase mitotic spindles were monitored by immunofluorescence and typical examples are given, endogenous Chk2 (red); kinetochores, CENP-H (green); chromosomes are stained with Hoechst 33342 (blue). Chk2 co-localization with chromosome structures was quantified in metaphases (mean $\pm S D ; n \geq 300$ metaphases) B) HuSOgen that express low levels of protein Chk2 were stably transfected with HA-tagged Chk2 and asynchronously growing cells were evaluated for lagging chromosomes; typical examples of lagging chromosomes containing anaphase and telophase are given (HA-Chk2, red; CENP-H, green; chromosomes, Hoechst 33342, blue). C) Asynchronously growing HuS30gen 
cells expressing H2B-GFP (to visualize in vivo lagging chromosomes) were analyzed by live cell microscopy. Left, phase-contrast images; middle, fluorescence images; right, merge. Scale bar, $10 \mu \mathrm{m}$. D) Asynchronously growing cells, HCT116, Huh7, HuSOgen, HuS30gen, and HuSOgen stably transfected with HA-tagged Chk2 (HuS-Chk2) were stained with CENP-H (constitutive component of kinetochores) and Hoechst 33342 (chromosomes). Anaphase and telophase lagging chromosomes were counted by immunofluorescence, (mean $\pm S D ; n=500$ cells) ${ }^{*} p<0.01$ vs HuSOgen. ${ }^{* *} p<0.05$ vs HuS-mock

Figure 5. Overexpressed/phosphorylated Chk2 is correlated with increased chromosomal missegregation. A) HuS30gen cells were stably retrovirally-transduced either with a control shRNA (shCTL) or shRNAs targeting CHK2 (shChk2). Chk2depleted HuS30gen cells were used to reconstitute expression of CHK2 by introducing a shRNA resistant mutant of CHK2 (myc-Chk2resist). The protein levels were determined by immunoblotting and a representative example is shown. B) Asynchronously growing cells HuS30-shCTL, HuS30-shChk2 and Chk2-depleted HuS30gen reconstituted with shRNA resistant mutant of CHK2 were stained with CENP-H (kinetochores) and Hoechst 33342 (chromosomes). Anaphase and telophase lagging chromosomes counted by immunofluorescence, (mean $\pm S D ; n=500$ cells), * $p<$ 0.01 vs controls. C) The mitotic index of asynchronously growing cells HuS30-shCTL, HuS30- shChk2 and Chk2-depleted HuS30 reconstituted with shRNA resistant mutant of $\mathrm{CHK} 2$ was measured by counting the mitotic figures (prophase, metaphase, anaphase, and telophase) positively stained for phosphorylated histone $\mathrm{H} 3(\mathrm{pHH} 3)$. The mitotic index was calculated as percentage of mitotic cells/total cells as described in Materials and Methods. Error bars show the SD from the means of three independent experiments, ${ }^{*} p<0.01$ vs controls. D) Chk2 phosphorylation levels on Thr68 and Thr387. Representative immunoblot out of three showing asynchronously growing HCT116, HuSOgen, HuS30gen, and HuSOgen stably transfected with Chk2 (HuS-Chk2). Cell lysates were immunoblotted against p-Thr68-Chk2 and p-Thr387-Chk2. E) Activated Chk2 localizes with mitotic structures in HuS30gen cells. Example of metaphase, anaphase and telophase from unstable HuS30gen cells and stable HCC cell line, 
Huh7. Activated Chk2 (p-Thr68-Chk2, red) co-localizes with CENP-H, constitutive component of kinetochores (green) only in HuS30gen. Arrowheads indicate lagging chromosomes, (Hoechst 33342, blue). Scale bar, $10 \mu \mathrm{m}$.

\section{Figure 6. Up-regulated Chk2 cooperates with Aurora B for chromosome condensation and cytokinesis}

A)HuSOgen cells showing expression and localization of endogenous Chk2 (red), Aurora B kinase (green), and chromosomes (Hoechst 33342, blue) B) Co-localization of Aurora B kinase (green) with HuSOgen overexpressing HA-tagged Chk2 (red) in mitoses with lagging chromosomes (Hoechst 33342, blue). Cells were fixed and analyzed by fluorescence microscopy. Arrows indicate spindle midzone region, arrowheads indicate midbody region. C) HuSOgen cells overexpressing HA-tagged Chk2 were stained with antibody anti-HA (red) and phospho-Histone H3 (green) to show co-localization of Chk2 with missegregating chromosomes. Scale bar, $10 \mu \mathrm{m}$.

Figure 7. FHA domain is essential for Chk2 localization and contributes to sustain lagging chromosomes. Red fluorescent protein was conjugated at the $\mathrm{N}$ terminus of each Chk2 mutant. Left, schematic diagrams. RFP was omitted. RFP-Chk2 mutants were transfected in HuS30gen cells, synchronized and blocked in metaphase by treatment with MG132 for 2 hours, the cells were analyzed by fluorescence microscopy. A) Expression and subcellular localization of RFP-Chk2. RFP-Chk2 decorates the spindle poles (two red dots), and localizes with kinetochores (CENP-H, green), chromosomes are stained with Hoechst 33342, blue. B) RFP-Chk2D347A, kinase dead mutant (KD), localizes similarly to RFP-Chk2. C) RFP-Chk2 $\triangle F H A$ does not localize to mitotic structures. D) RFP-FHA localizes to spindle poles, kinetochores. FHA domain tagged with RFP is similar to RFP-Chk2. Scale bar, $10 \mu \mathrm{m}$. E) Quantification of lagging chromosomes during anaphases and telophases in HuSOgen cells stably expressing Chk2(KD), Chk2 $\mathrm{FHA}$ and Chk2FHA mutants. Chk2 FHA mutant significantly reduces the number of lagging chromosomes as a result of the dominant 
negative effect towards endogenous Chk2. mean $\pm S D ; n=400$ anaphases and telophases. ${ }^{*} p<0.01 \mathrm{vs}$ control

Figure 8. P53 and phosphorylated Rb expression in HCT116, HuS30gen and HuS0gen. A) Representative immunoblot out of three showing the protein levels of p53 in HCT116, Huh7, HuS30gen and HuS0gen cells. In the same blot are represented p53 expression levels of HuSOgen cells after treatment with DNA-damaging agent 5fluorouracil (5-FU, 1 $\mu \mathrm{M})$. B) Representative images of HCT116, Huh7 and HuS30gen cells fixed and stained with an antibody directed against the mutated codon TGT at position 220 of p53 protein. Huh7 cells, previously characterized as p53 mutated (see reference 30), are used as positive control. C) Representative immunoblot out of three independent experiments showing the protein levels of $\mathrm{Rb}$ and phosphorylated $\mathrm{Rb}, \mathrm{p}-\mathrm{Rb}$ (Ser807/811), in HCT116, HuS30gen and HuSOgen cells. D), E) and F) Mitoses of asynchronously growing HCT116, HuS30gen and HuSOgen cells stained with the antibody specific for phosphorylated $\mathrm{Rb}$ and analyzed by fluorescence microscopy; p-Rb (Ser807/811) red; chromosomes (Hoescht 33342, blue). Scale bar, $10 \mu \mathrm{m}$.

Figure 9. A proposed schema illustrating the mechanism by which Chk2 overexpression and mislocalization sustains defective mitoses throughout hepatic tumorigenesis. Mitotic errors represented by lagging chromosomes cause DNA damage and overexpress Chk2 in genetically unstable cells. Chk2 and phosphorylated forms of Chk2 mislocalize within the mitotic structures in the presence of DNA damage contributing to increase the numbers of lagging chromosomes. 


\title{
CHK2 Overexpression and Mislocalization within Mitotic Structures Enhances Chromosomal Instability and Hepatocellular Carcinoma Progression
}

Vinicio Carloni ${ }^{1}$,Matteo Lulli ${ }^{2}$, Stefania Madiai ${ }^{1}$, Tommaso Mello ${ }^{3}$, Andrew Hall ${ }^{4}$,Tu Vinh Luong $^{4}$, Massimo Pinzani ${ }^{1,5}$, Krista Rombouts ${ }^{1,5^{*}}$ and Andrea Galli ${ }^{3^{*}}$

${ }^{1}$ Department of Experimental and Clinical Medicine, ${ }^{2}$ Department of Experimental and Clinical Biomedical Science, General Pathology Unit, ${ }^{3}$ Department of Experimental and Clinical Biochemical Sciences, Gastroenterology Unit, University of Florence, Florence, Italy, ${ }^{4}$ Royal Free Hospital, Department of Cellular Pathology, London, UK, ${ }^{5}$ University of London (UCL)Institute for Liver \&Digestive Health, London, United Kingdom MP and KR have relocated to the University of London, London, UK * These authors contributed equally.

Correspondence should be addressed to: Vinicio Carloni MD., PhD, Department of Experimental and Clinical Medicine. University of Florence, Largo Brambilla 3, 50134, Florence, Italy. Phone:+39-55-2758091; Fax:+39-55-7947104; E-mail: vinicio.carloni@unifi.it

Key words: DNA damage response proteins, DNA double-strand breaks, genomic instability, defective chromosome segregation, DNA damage

\begin{abstract}
Abbreviations list: CIN, chromosomal instability; Chk2, checkpoint kinase 2; FHA, forkhead-associated domain; SCD, SQ/TQ cluster domain; hTERT, human telomerase reverse transcriptase; Nek2, NIMA related kinase 2; CENP-H, centromere protein $\mathrm{H}$; $\mathrm{pHH}$, phospho-Histone $\mathrm{H} 3$; Rb, retinoblastoma protein; HA, hemagglutinin; RFP, red fluorescent protein.
\end{abstract}

Count Words: 3938 


\title{
Significance of this study
}

\author{
What is already known on this subject?
}

- Chromosomal instability (CIN), is a major hallmark of hepatic tumorigenesis and hampers prognosis and therapy. The underlying mechanisms are not well characterized.

- $\quad$ IN is regarded as an ongoing elevated frequency of mitotic errors that leads to numerical and structural chromosome aberrations in a significant proportion of cell divisions leading to intra-tumoral heterogeneity and drug resistance.

- DNA damage caused by genotoxic agents activates DNA damage response checkpoint kinase 2 (Chk2) protein expression

- Mislocalization of proteins, including oncoproteins, tumor suppressors, and other cancer-related proteins, can interfere with normal cellular function and cooperatively drive tumor development

What are the new findings?

- Mitotic errors represented by lagging chromosomes cause DNA damage and overexpress Chk2 throughout hepatic tumorigenesis

- Chk2 and the activation/phosphorylated forms of Chk2 mislocalize within the mitotic structures in the presence of DNA damage contributing to increased numbers of cells with lagging chromosomes. In contrast, knockdown of Chk2 reduces the number of cells with lagging chromosomes and the mitotic index

- The Chk2 forkhead-associated domain (FHA) is responsible for the mislocalization of the protein at the mitotic structures

- Chk2 is expressed at the nuclear level in a subset of human HCC and this aspect correlates with HCC progression. Moreover, CHK2 expression is significantly higher in HCC patients with mutated TP53, a hallmark of genomic instability, than in those with mutated CTNNB1/beta-catenin.

How might it impact on clinical practice in the foreseeable future?

- The study reveals a new mechanistic insight in the co-involvement of Chk2 in CIN i.e. Chk2 mislocalization, hence indicating the possibility to use Chk2 as a new drug target in $\mathrm{HCC}$ 
- The findings of this study suggest Chk2 as a putative biomarker that can be used to detect DNA damage and chromosomal instability in HCC providing a valuable support, not only for prognosis, but also for identifying patients who are likely to have a response to therapy. 


\begin{abstract}
Objective

Chromosomal instability (CIN) is the most common form of genomic instability, that promotes hepatocellular carcinoma (HCC) progression by enhancing tumour heterogeneity, drug resistance and immunity escape. CIN per se is an important factor of DNA damage, sustaining structural chromosome abnormalities but the underlying mechanisms are unknown.
\end{abstract}

\title{
Design
}

DNA-damage response protein Chk2 expression was evaluated in an animal model of diethylnitrosamine-induced HCC characterized by DNA damage and elevated mitotic errors. Chk2 was also determined in two discrete cohorts of human HCC specimens. To assess the functional role of Chk2, gain on- and loss-of-function, mutagenesis, karyotyping, and immunofluorescence/live imaging were performed by using HCT116, Huh7 and human hepatocytes immortalized with hTERT gene (HuS).

\section{Results}

We demonstrate that mitotic errors during HCC tumorigenesis cause lagging chromosomes/DNA damage and activation of Chk2. Overexpression/phosphorylation and mislocalization within the mitotic spindle of Chk2 contributes to induce lagging chromosomes. Lagging chromosomes and mitotic activity are reversed by knockdown of Chk2. Furthermore, up-regulated Chk2 maintains mitotic activity interacting with Aurora B kinase for chromosome condensation and cytokinesis. The forkheadassociated (FHA) domain of Chk2 is required for Chk2 mislocalization to mitotic structures. In addition, retinoblastoma protein phosphorylation contributes to defective mitoses. A cohort and independent validation cohort show a strong cytoplasm-to nuclear Chk2 translocation in a subset of HCC patients.

\section{Conclusions}

The study reveals a new mechanistic insight in the co-involvement of Chk2 in HCC progression. These findings propose Chk2 as a putative biomarker to detect CIN in HCC providing a valuable support for clinical/therapeutical management of patients. 


\section{Introduction}

Hepatocellular carcinoma $(\mathrm{HCC})$ is the primary malignancy of hepatocytes and the most frequent solid tumour of the liver. Half a million cases occur annually and the World Health Organization has recently ranked $\mathrm{HCC}$ as the second most lethal cancer worldwide [1]. Different etiological factors such as hepatitis B and C virus, alcohol and diabetes cause liver injury followed by inflammation, necrosis and hepatocytes proliferation [2]. Continuous cycles of this destructive-regenerative process culminates in liver cirrhosis which is characterized by regenerating nodules that progress to dysplastic nodules and ultimately $\mathrm{HCC}[3,4]$. More than $90 \%$ of $\mathrm{HCC}$, including dysplastic nodules in cirrhotic liver, possess chromosomal aberrations suggesting that chromosomal defects occur at early stages of tumour development and several attempts have been made to connect different chromosomal aberration patterns as specific subsets of HCC, albeit with limited success [5-7]. A common feature of human $\mathrm{HCC}$ and dysplastic nodules is represented by chromosomal instability (CIN). Various types of chromosomal aberrations, including numerical (aneuploidy) and structural (e.g., translocations, deletions), are found in $\mathrm{HCC}$ and are linked to tumorigenesis [8,9]. An important aspect in studying HCC tumorigenesis is to distinguish between the state of the karyotype, and the rate of karyotypic change. In fact, aneuploidy is not synonymous with CIN, because aneuploid tumours are stable with uniform karyotype and phenotype [10-12]. Conversely, there is an increased rate of CIN generating diverse karyotypes within the same tumour. This yields a heterogeneous tumour cell population that has the ability to undergo selective evolution in term of drug resistance and immune system escape mechanisms [13-15]. CIN is regarded as an ongoing elevated frequency of mitotic errors that leads to additional chromosome gains or losses in a significant proportion of cell divisions. Our hypothesis is that CIN per se is also an important mechanism of DNA damage inducing structural chromosome aberrations in HCC. To verify this hypothesis we utilized checkpoint kinase 2 (Chk2) as a central effector of the cell response to DNA damage. In fact, Chk2 has been described to detect a variety of DNA lesions caused by exogenous and endogenous genotoxic agents. Our aim is to investigate the expression of this kinase in hepatic carcinogenesis and its correlation with the genesis of structural chromosomal 
aberrations. Here, we provide evidence that defective chromosome segregation i.e. lagging chromosomes causes DNA damage and determines an overexpression/phosphorylation of Chk2. Furthermore, the mislocalization of Chk2 with components of the mitotic spindle contributes to chromosome missegregations and DNA damage. These data are corroborated with the observation of an increased Chk2 and nuclear translocation in two independent cohorts of human HCC specimens. 


\begin{abstract}
Materials and Methods
Please refer to the Supplementary Materials and Methods for more detailed descriptions.
\end{abstract}

\title{
Tissue Samples and Immunohistochemistry Assays.
}

Florence cohort, University Hospital of Florence; formalin-fixed, paraffin-embedded tissue were obtained from an archive of liver biopsies of patients diagnosed with HCC. H\&E-stained tissue sections of 49 patients were reviewed, and areas of cirrhotic and HCC were identified. Chk2 antigen retrieval was performed by heating in citrate buffer $\mathrm{pH} 6.0$ at $98^{\circ} \mathrm{C}$ for 30 minutes. Non-specific signal was eliminated by peroxidase block for 10 minutes at RT. Primary antibody was incubated at room temperature overnight in a humidified chamber followed by HRP-conjugated secondary antibody incubation for 30 minutes at room temperature. Antibody binding was revealed by DAB and reaction was stopped by immersion of tissue sections in distilled water once brown colour appeared. Tissue sections were counterstained by hematoxylin, dehydrated in graded ethanols and mounted. All reagents for immunohistochemistry were from Dako. Appropriate positive and negative controls were included for each run of immunohistochemistry. Chk2 expression was determined according to $\mathrm{H}$-score method. For $\mathrm{H}$-score assessment, 10 fields were chosen at random at $\times 400$ magnification and the staining intensity in the malignant cell nuclei was scored as $0,1,2$, and 3 corresponding to the presence of negative, weak, intermediate, and strong brown staining, respectively. The total number of cells in each field and the number of cells stained at each intensity were counted. The average percentage positive was calculated, and a $\mathrm{H}$-score between 0 and 300 was obtained where 300 was equal to $100 \%$ of tumor cells stained brown strongly. The positive cut off was considered $\geq 100$ (supplemental Figure S1). Chk2 expression in biopsy sections was scored by a pathologist and two researchers independently.

London cohort, Royal Free Hospital; formalin-fixed, paraffin-embedded surgical specimens from consecutive patients diagnosed with HCC were retrieved from Royal Free Hospital archives. All samples were stained with H\&E and the sections were 
reviewed and suitable areas of cirrhosis and HCC were identified in 20 samples. Antigen retrieval was performed by microwaving $(720 \mathrm{~W})$ in $\mathrm{pH} 6.0$ sodium citrate buffer for 30 mins. Endogenous peroxidase and non-specific binding of primary antibody was blocked. The primary Chk2 antibody was incubated at overnight at $5^{\circ} \mathrm{C}$. Primary antibody detection was carried out using the Novolink polymer detection system (Novocastra).Tissue sections were counterstained with Mayer's hematoxylin.

Experimental hepatocarcinogenesis.Twenty-five female Wistar rats (210-225 g) were given diethylnitrosamine $50 \mathrm{mg} / \mathrm{L}$ ad libitum in their drinking water for a period of 10 weeks as previously described [16]. Another group of five animals were maintained as controls. All animals were administered a standard laboratory diet and were weighed each week. Five animals for each time point were sacrificed on week 4, 6, 8, 10 and, 14.Tumours and liver tissues were removed and fixed in 10\% neutral buffered formalin. Liver sections $(5 \mu \mathrm{m}) \quad$ were cut from paraffin-embedded blocks and stained Chk2 (Abcam, \#ab47433), phospho-Histone H2A.X (Cell Signaling, \#9718) and hematoxylin and eosin (H\&E) for histological examination and expression were determined according to $\mathrm{H}$-score method.

\section{CHK2 Expression in HCC samples from Cancer Genome Atlas (TCGA) database.}

The TCGA-LIHC cohort, containing 377 cases, was used to validate CHK2 expression levels in distinct subtypes of HCC. The TCGA database (https://gdc-portal.nci.nih.gov) was accessed on November 16, 2016 through the cGbioportal website (http://www.cbioportal.org/) to retrieve patients with either TP53 or CTNNB1 mutation. The TCGA_participant_barcode was used to download CHK2 expression from RNA-seq data using the firebrowse portal (http://firebrowse.org). Patients with co-occurrent mutations of TP53 and CTNNB1 were excluded from the analysis. CHK2 expression levels were retrieved for 101 patients with TP53 mutation and 85 patients with CTNNB1 mutation. Statistical analysis was performed using the Mann-Whitney test with GraphPad 6 software. 


\section{Results}

\section{Chk2 is overexpressed in a subset of human HCC}

To test our hypothesis that hepatic tumorigenesis per se could be a cause of DNA damage and structural chromosome aberrations, an animal model of diethylnitrosamineinduced HCC was employed which induced macroscopical visible tumours after 14 weeks (Figure 1A). This model has previously been established to induce elevated mitotic errors, DNA damage and chromosome aberrations [17]. Using this model we can compare the expression levels of DNA damage response proteins induced by the genotoxic activity of diethylnitrosamine with a later phase represented exclusively by tumorigenesis. Therefore, Chk2, a DNA damage-response checkpoint kinase, and phospho-Histone H2A.X (Ser139) a component of chromatin which becomes phosphorylated following DNA damage (e.g. DNA double-stranded breaks) were investigated [18]. As shown in Figure 1B several nuclei of cancer cells exhibited an increased expression of Chk2 and phospho-Histone H2A.X revealing the presence of DNA damage. A timeline analysis of liver sections demonstrated a two-fold increase in Chk2 basal levels at 4 weeks followed by decreased values after 8 weeks (Figure 1C). In contrast, by 14 weeks tumours were macroscopically visible and histologically evident in $80 \%$ of animals. Immunostaining of HCC sections showed a significant increased expression of Chk2 and phospho-Histone H2A.X (Figure 1C and 1D) when compared with liver sections without evidence of tumours (8 weeks). Taken together, these data showed that the increased expression of Chk2 reflected a response triggered by DNA damage during tumorigenesis.

To extend these findings to humans, the expression of Chk2 in patients with cirrhoticrelated HCC and normal liver was examined in liver biopsies from hospital archives (Florence cohort, supplemental Table 1). A nuclear staining of Chk2 was detectable in distinctive histotypes of HCC (supplemental Figure S2 and S3). More specifically, the expression level of Chk2 in different grades of HCC was evaluated. Remarkably, within histological grade II and III, significant quantitative differences between positive and negative Chk2 patients were found $(p<0.001)$. Overall, these data demonstrated that 
Chk2 nuclear positivity was found in $63.2 \%$ (31 of 49) of HCCs analyzed (supplemental Table 2 ). A separate validation cohort of patients was examined at Royal Free Hospital, London (supplemental Table 3). Normal liver tissue showed a moderate and diffuse cytoplasmic staining, whereas Grade 1 specimens were characterized with weak focal cytoplasmic Chk2 staining. In Grade 2, a Chk2 perinuclear and nuclear accumulation was observed. Specimens with Grade 3 showed exclusively a clear and strong nuclear staining, without cytoplasmic and perinuclear Chk2 staining (Figure 2a and supplemental Table 4). Furthermore, the expression levels of $\mathrm{CHK} 2$ were analyzed in a public available cohort of HCC from the TCGA database. We found a highly-significant increase of CHK2 expression in HCC with mutated TP53, a hallmark of genomic instability, with respect to those with CTNNB1/beta-catenin mutation (Figure 2b).

\section{Defective chromosome segregations cause DNA damage}

The previous observations prompted us to investigate the functional role of Chk2. For this reason, three different cell lines were employed. The colon carcinoma cell line HCT116 was used i.e. a near-diploid cell line that faithfully segregates chromosomes to maintain a stable karyotype and is commonly employed in CIN studies [19,20]. Secondly, the HCC cell line Huh7 was used which is a stable hyperdiploid karyotype with altered chromosomal structures and finally HuS cells were used marked with an unstable karyotype [21]. HuS cells are human hepatocytes immortalized with human telomerase reverse transcriptase (hTERT), a gene described commonly activated in human HCC $[8,9]$. HuS cells just established in culture ( 0 generation) and hereafter referred to as HuSOgen showed a karyotype with a distribution of 46 chromosomes (Figure 3A) and expressed low levels of Chk2 protein in comparison to HCT116 and Huh7 (Figure 3B). The HuSOgen cells were maintained in culture and after 30 consecutive generations (HuS30gen) were re-examined to verify the karyotype status. The cells showed a distribution near-triploid, with many structural chromosomal defects (Figure $3 \mathrm{C}$ and supplemental Figure S4A) and importantly this aspect was associated with increased expression of Chk2 protein, (Figure 3B). Likewise with the murine model, we evaluated the expression levels of phospho-Histone H2A.X (Ser139) in all 
the cell lines studied. As shown in Figure 3D, both HuSOgen and HuS30gen cells exhibited high levels of phospho-Histone H2A.X, although with different levels of expression. These findings indicate the presence of DNA double-strand breaks, a hallmark of DNA damage, in HuS cells.

Overexpressed Chk2 mislocalizes with mitotic structures in HuS30gen cells but not in HCT116 and Huh7.

The finding of overexpressed Chk2 in both karyotyped stable HCT116 and Huh7 and in unstable karyotyped HuS30gen did not demonstrate a specific role for Chk2 in defective chromosome segregation. Therefore, we analyzed the cell lines HCT116, Huh7, HuSOgen and HuS30gen by immunofluorescence to examine a possible altered localization of Chk2 with the mitotic structures. Chk2 showed a localization to the spindle poles of metaphase mitotic structures in HCT116, Huh7, and HuS0gen (Figure 4A). Very importantly, HuS30gen cells showed a robust localization of Chk2 with all metaphase mitotic structures indicating a clear mislocalization of Chk2 (Figure 4A and supplemental Figure S4B). These results suggest that in HuSOgen cells, expressing low levels of DNA damage, Chk2 weakly co-localized with the mitotic spindle components, whereas Chk2 is increased and strongly associated with all mitotic structures in HuS30gen cells that are expressing high levels of DNA damage. To further address these findings we stably transfected HuSOgen with HA-tagged Chk2, HuSOgen overexpressing Chk2 exhibited Chk2 localization with mitotic structures as shown in Figure 4B. Next, we measured the number of lagging chromosomes (Figure 4C) in HuSOgen, HuS30gen, and in HuSOgen stably transfected with HA-tagged Chk2 to mimic overexpression of Chk2. As shown in Figure 4D, overexpression of Chk2 significantly correlated with high number of mitoses with lagging chromosomes in HuS30gen, and in HuSOgen overexpressing HA-tagged Chk2. These data clearly indicate that indeed overexpression and mislocalization of Chk2 itself contributes to defective chromosome segregations. Furthermore, to assess that the increased expression of Chk2 was specifically related to those mitoses with defective chromosome segregations and not a random deregulation of proteins, the expression of Nek2 (NIMA related kinase 2) protein was evaluated. Nek2 is a protein located at the 
centrosome/spindle poles and responsible for chromosomal defective segregations [22]. HCT116 and Huh7 cells showed a strong Nek2 expression whereas both HuSOgen and HuS30gen cells expressed low levels of Nek2 (supplemental Figure S5) suggesting that the previously observed overexpression of Chk2 in Hus30gen was a specific event that coincided with structural chromosomal defects and DNA damage.

\section{Chk2 overexpression is correlated with increased chromosomal missegregations} The findings that HuS30gen express high levels of Chk2 protein correlating with DNA damage (Figure 3B and $3 \mathrm{D}$ ), prompted us to investigate the effects of knocking down expression of CHK2 in HuS30gen cells by retroviral-mediated short hairpin RNA (shRNA) transduction. A pool of four shRNA targeting Chk2 resulted in effective decrease of the protein (Figure $5 \mathrm{~A}$ ). It is important to note that silencing CHK2 coincided with a significant reduction of the number of HuS30gen cells with lagging chromosomes and HuS30gen mitotic index. To address any off target effects of the hairpins, we rescued the mitotic index and the number of cell with lagging chromosomes of the knockdown HuS30gen by expressing a myc-tagged version of Chk2 that circumvents the shRNA directed against human Chk2 (Figure 5B and 5C). These data demonstrate that Chk2 overexpression is correlated with increased chromosomal missegregation and mitotic index.

\section{Defective mitoses-related DNA damage promotes Chk2 phosphorylation and mislocalization within mitotic structures.}

Human CHK2, is a 543 amino acid protein that consists of a N-terminal SQ/TQ cluster domain (SCD), a central forkhead-associated (FHA) domain, and a C-terminal serine/threonine kinase domain [23]. The SCD consists of multiple SQ/TQ (Ser-Gln/ThrGIn) motifs with Thr68 being the primary site to be phosphorylated in response to DNA damage followed by phosphorylation of Thr387 in the activation loop of the kinase domain $[24,25]$. Therefore, the protein expression of phospho-Thr68-Chk2 and phospho-Thr387-Chk2 in HuS30gen and HuS0gen stably transfected with HA-tagged 
Chk2 were assessed. As shown in Figure 5D phospho-Thr68-Chk2 and phosphoThr387-Chk2 were highly expressed in HuS30gen and in HuSOgen stably transfected with HA-Chk2, but not in HuS0gen as expected. Furthermore, phospho-Thr68-Chk2 revealed a robust localization with components of the mitotic spindle as previously shown for Chk2. In particular, a prominent association of phospho-Thr68-Chk2 was observed with mitotic structures such as the spindle poles, kinetochores and midbody in Hus30gen cells, (Figure 5E, upper pane/s). In contrast, Huh7 cells expressing low levels of DNA damage ( see Figure 3D) did not show co-localization of phosphoThr68-Chk2 with mitotic structures, (Figure 5E, lower panels). Taken together, these results reveal an overexpression/mislocalization of phosphorylated Chk2, which is correlated with its activation status, and manifestations of lagging chromosomesinduced DNA damage.

\section{Up-regulated Chk2 cooperates with Aurora B for chromosome condensation and cytokinesis.}

As previously shown the typical localization of activated Chk2 in HuS30gen cells was observed in kinetochores and specifically the midbody, suggesting a possible interaction with Aurora B kinase. This protein is known to be involved in chromosome condensation, the assembly of the spindle midzone in anaphase and the midbody during telophase. Furthermore, Aurora B kinase has been identified as an effective predictor of aggressive $\mathrm{HCC}$ recurrence, in relation to genomic instability [26]. Therefore, HuSogen cells were transfected with HA-tagged Chk2 and immunofluorescence analyses revealed a co-localization of Chk2 with Aurora B throughout all mitotic phases, and this co-localization was also present in cells marked with lagging chromosomes (Figure $6 \mathrm{~A}$ and $6 \mathrm{~B}$ ). Aurora $\mathrm{B}$ is responsible for the phosphorylation of histone $\mathrm{H} 3(\mathrm{pHH} 3)$, and $\mathrm{pHH} 3$ serine residue (Ser-10) is considered to be a crucial event for the onset of mitosis. The utility of $\mathrm{pHH} 3$ to identify mitoses has been demonstrated in several cancer studies [27-29]. As, the co-localization of Aurora B with Chk2 was demonstrated we further explored the co-localization of overexpressed HA-Chk2 with pHH3. As shown in Figure 6C, Chk2 exhibited a striking co-localization 
with $\mathrm{pHH} 3$. Overall, the data so far demonstrate that in unstable HuS cells during mitosis, lagging chromosomes cause DNA damage, overexpression of Chk2 which localizes to all mitotic structures. This overexpressed and mislocalized Chk2 protein contributes to a constant mitotic activity and more important Aurora B kinase and $\mathrm{pHH} 3$ interact with Chk2. This favours a possible scenario where an overexpressed and mislocated Chk2 overrules the signalling specificity at distinct mitotic checkpoints inducing itself chromosomal aberrations, hence CIN.

\section{The forkhead associated domain (FHA) determines Chk2 mislocalization to mitotic structures and is essential for DNA damage response}

To understand how Chk2 is recruited to the mitotic structures in such a specific manner, we investigated Chk2 specific domain(s) that are required for Chk2 localization. A series of RFP-tagged Chk2 variants were created, each variant carrying a point mutation or a deletion, and were expressed and localized individually in HuS30gen. The RFP-Chk2D347A, a kinase-inactive mutant, localized similarly as shown for RFP-Chk2 (Figure 7A). This indicated that the kinase activity is dispensable for Chk2 mislocalization (Figure 7B). The FHA domain of Chk2 has been proposed to mediate localization of Chk2 to mitotic structures for the reason that FHA domains function as phospho-threonine-binding protein motifs. The deletion of the Chk2 FHA domain disrupted the localization of the protein to mitotic structures such as the spindle poles and kinetochores indicating that the deletion of the FHA is responsible for the observed mislocalization (Figure 7C). Indeed, the entire FHA domain RFP-tagged localized to mitotic structures similar to RFP-Chk2 (Figure 7D). Along these lines, we can conclude that the FHA domain is required for proper Chk2 localization to mitotic structures. Furthermore, we analyzed the effects of the several Chk2 mutants on lagging chromosomes. Interestingly, transfection of HuSOgen with Chk2 FHA domain significantly reduced the number of lagging chromosomes (Figure 7E). This result suggests a dominant negative effect of Chk2 FHA domain towards endogenous Chk2.

Rb phosphorylation contributes to defective mitoses in HuS cells. 
An important point raised by defective mitoses-related DNA damage as observed in this study is why the cells do not sense signals deployed to curb cell proliferation, as normally the growth of cultured diploid cells is halted by chromosome missegregation. In HCC, p53 deficiency has been described to participate in the development of CIN, however the relevance of $\mathrm{p} 53$ to $\mathrm{HCC}$ initiation, progression, or both, remains an area of active investigation [2]. Therefore, the basal level of p53 in HCT116, Huh7, HuS30gen and HuSOgen was investigated. As shown in Figure 8A, p53 was expressed in Huh7 and at low level in HuS30gen cells. HuSOgen cells did not express p53 in basal conditions, however treatment of the cells with 5-fluorouracil, a DNA-damaging agent, triggered a strong p53 protein expression. Based on these findings we assume that p53 expression may be a late event in HuS cells, as a result of several defective mitoses [19]. Furthermore, mutated p53 was not present in HCT116 and HuS30gen cells in contrast to Huh7 cells that we used as a positive control (Figure 8B) [30].

The possible involvement of the retinoblastoma protein $\mathrm{Rb}$ was evaluated since several studies have described $\mathrm{Rb}$ as an important regulator of cell division [31]. Specifically, $\mathrm{Rb}$ phosphorylation determines $\mathrm{Rb}$ inactivation and leads to defective mitoses [31]. Therefore, we evaluated the protein expression levels of phospho-Rb (Ser807/811) in HCT116, HuSOgen cells and HuS30gen. As shown in Figure 8C HuS30gen cells express higher levels of phospho-Rb (Ser807/811) in comparison to HuSogen cells. Moreover, HCT116 (Figure 8D), HuS30gen (Figure 8E) and HuSOgen (Figure 8F) exhibited a localization of phospho-Rb (Ser807/811) at the chromosomal level. Taken together, these findings suggest that in HuS30gen $\mathrm{Rb}$ phosphorylation is required to sustain mitotic activity which leads to mitotic defects. 


\section{Discussion}

The neoplastic evolution of HCC proceeds through a multi-step histological process that is less well defined than that of other cancer types. Hyperplastic nodules of regenerating hepatocytes have normal karyotype and represent a potential first step towards HCC. These lesions can progress to dysplastic nodules, which have abnormal chromosome features including numerical and structural alterations. These dysplastic nodules can evolve to HCC marked with recurrent regions of copy number change and allelic imbalances [3, 4]. Loss of certain tumour suppressors or gain of specific oncogenes promote cell division as shown in many previous studies [25, 32]. We demonstrate that this coincides with defective mitoses which can cause DNA damage. This scenario is conceivable considering that massive deregulation of mitosis is incompatible with cell survival and can even be tumour suppressive [33]. Therefore, subtle rather than massive mitotic defects are expected to underlie CIN in HCC, allowing a tolerable level of chromosome defects. To support this view we have provided evidence in this study that hepatocarcinogenesis with lagging chromosomes elicits the expression of DNA damage response protein Chk2. Thus, the overexpression of Chk2 and its mislocalization within structures of the mitotic spindle sustains cell division and chromosomes missegregation [34-36]. This forms a self-perpetuating mechanism causing DNA damage (Figure 9).

We have also found that functional $\mathrm{Rb}$ inactivation is necessary but not sufficient to trigger chromosome missegregation. Indeed, phosphorylation of $\mathrm{Rb}$ deregulates transcription factor E2F, and well-characterized targets of E2F include multiple genes whose products are required to maintain cell division [37-39]. The integrity of chromosomal DNA is under constant surveillance throughout the cell division. Surprisingly, the fact that a deregulated functional Chk2 itself might cause per se CIN through abnormal spindle localization is intriguing, as the role of Chk2 in the maintenance of genetic stability has mainly been ascribed to its function in DNA damage checkpoint signalling.

When DNA damage occurs, Chk2 is first activated in the nucleus during interphase, spreads to the cytoplasm and localizes to the various mitotic structures because of the 
phosphopeptide-binding ability of the FHA domain [40]. During DNA damage, chromosomes accumulate a number of phosphoproteins around DNA damage sites [41]. Chk2 may bind to specific phosphoproteins through the FHA domain forming numerous chromosomal foci and trap Chk2 to the structures. When this FHA domain is deleted, the molecule is excluded from the mitotic chromosomes and therefore does not mislocalize to kinetochores, spindle poles and midbody as was demonstrated in Figure 7. Moreover, Chk2 localization to the kinetochores and midbody region suggests that Chk2 may directly affect spindle assembly function, similar to Aurora B kinase [42], but only when DNA damage exists. The data obtained in this study fit a model whereby abnormal expression of Chk2 and Aurora B kinase disrupts the sensitive balance of mitotic proteins, which in turn undermines faithful chromosome segregation [34]. If the relative expression levels of the proteins involved in the quality control of the machinery which ensures chromosome segregation fidelity is disrupted by mitotic errors and DNA damage, the resulting imbalance could further compromise chromosome segregation accuracy.

Since Chk2 overexpression can induce oligomerization and cis- and trans phosphorylation of regulatory residues $[43,44]$, one possibility is that local enrichment of Chk2 at kinetochores and midbody regions facilitates Chk2 oligomerization and activation. This mechanism may alter regulation of Chk2-dependent processes such as centrosome function, cell cycle progression, and chromosome segregation. The discovery of a role for Chk2 in HCC, its association with DNA damage and CIN may have potential diagnostic and prognostic significance. Importantly, grading heterogeneity inside a tumour is frequently observed and may significantly limit diagnosis and choice of treatment. This study suggests that stratification of HCC patients according to CIN status and/or DNA damage may be useful to minimize the confounding effects of tumor CIN status in clinical trials. Along these lines, we propose that the diagnostic and prognostic value of Chk2 should be further verified in prospective clinical trials. 
Competing interests: The authors have declared that no conflict of interest exists.

Contributors: V.C. study concept and experimental design, cloning experiments, analysis and interpretation of data, drafting of the manuscript, study supervision; M.L. immunoblotting and karyotype experiments; S.M. immunohistochemistry and animal experiments; T.M. immunofluorescence, live-cell, bioinformatics analysis; A.H. immunohistochemistry analysis; TV.L. human tissue evaluation; M.P. critical review of the manuscript; K.R. experimental design, data analysis, critical review of the manuscript; A.G conceptual and intellectual input, critical review of the manuscript. All authors read and approved the final manuscript.

Funding:This study was in part supported by the University of Florence and Regione Toscana "Ricerca regionale in materia di salute D.D. n. 3242 ", and by Ministero dell'Istruzione, dell'Università FIRB RBAP10MY35_002 and FiorGen Foundation. 


\section{References}

[1] McGlynn KA, Petrick JL, London WT. Global epidemiology of hepatocellular carcinoma: an emphasis on demo graphic and regional variability. Clin Liver Dis 2015;19:223-238

[2] Farazi PA, DePinho RA. Hepatocellular carcinoma pathogenesis: from genes to environment. Nat Rev Cancer. 2006;6:674-87.

[3] Carloni V, Luong T V, Rombouts K. Hepatic stellate cells and extracellular matrix in hepatocellular carcinoma: more complicated than ever. Liver Int. 2014;34:834-843.

[4] Mazzocca A, Liotta F, Carloni V. Tetraspanin CD81-regulated cell motility plays a critical role in intrahepatic metastasis of hepatocellular carcinoma. Gastroenterology 2008; 135:244-256

[5] Sakakura C, Hagiwara A, Taniguchi $\mathrm{H}$, et al. Chromosomal aberrations in human hepatocellular carcinomas associated with hepatitis $C$ virus infection detected by comparative genomic hybridization. Br. J. Cancer 1999;80: 2034-2039.

[6] Wong N, Lai P, Pang E, et al. A comprehensive karyotypic study on human hepatocellular carcinoma by spectral karyotyping. Hepatology 2000;32: 1060-1068.

[7] Kusano N, Shiraishi K, Kubo K, et al. Genetic aberrations detected by comparative genomic hybridization in hepatocellular carcinomas: their relationship to clinic pathological features. Hepatology.1999;29:1858-62

[8] Shimojima $M$, Komine $F$, Hisatomi $H$, et al. Detection of telomerase activity, telomerase RNA component, and telomerase reverse transcriptase in human hepatocellular carcinoma. Hepatol. Res. 2004; 29: 31-38.

[9] Youssef N, Paradis V, Ferlicot S, et al. In situ detection of telomerase enzymatic activity in human hepatocellular carcinogenesis. J. Pathol. 2001;194: 459-465.

[10] Kawai, H. Suda T, Aoyagi Y, et al. Quantitative evaluation of genomic instability as a possible predictor for development of hepatocellular carcinoma: comparison of loss of heterozygosity and replication error. Hepatology 2000; 31: 1246-1250.

[11] Guan XY, Fang Y, Sham JS, et al. Recurrent chromosome alterations in hepatocellular carcinoma detected by comparative genomic hybridization. Genes Chromosomes Cancer 2000; 29:110-116. 
[12] Wilkens L, Flemming $P$, Gebel $M$, et al. Induction of aneuploidy by increasing chromosomal instability during dedifferentiation of hepatocellular carcinoma. Proc. Natl Acad. Sci. USA. 2004;101: 1309-1314

[13] Almendro V, Marusyk A, Polyak K. Cellular heterogeneity and molecular evolution in cancer. Annu Rev Pathol. 2013;8:277-302.

[14] Bakhoum F S, Compton A D. Chromosomal instability and cancer: a complex relationship with therapeutic potential. J Clin Invest. 2012;122:1138-1143

[15] Carloni V, Mazzocca A, Mello T, et al. Cell fusion promotes chemoresistance in metastatic colon carcinoma. Oncogene. 2013; 32: 2649-266

[16] Carloni V, Vizzutti F, Pantaleo P. Farnesyltransferase inhibitor ABT-100 is a potent liver cancer chemopreventive agent. Clin Cancer Research. 2005; 11: 4266-4274.

[17] Basak R, Saha BK,Chatterjee M. Inhibition of diethylnitrosamine-induced rat liver chromosomal aberrations and DNA-strand breaks by synergistic supplementation of vanadium and 1a,25-dihydroxyvitamin D3. Biochim Biophys Acta. 2000;1502:273-282 [18] Rogakou EP, Pilch DR, Orr AH, et al. DNA double-stranded breaks induce histone H2AX phosphorylation on serine 139. J Biol Chem. 1998;273:5858-68

[19] Thompson SL, Compton DA. Proliferation of aneuploid human cells is limited by a p53-dependent mechanism. J Cell Biol. 2010; 188:369-381

[20] Stolz A, Ertych N, Kienitz A, et al. The CHK2- BRCA1 tumour suppressor pathway ensures chromosomal stability in human somatic cells. Nat Cell Biol. 2010 ;12:492-499.

[21] Durantel D, Zoulim F. Going towards more relevant cell culture models to study the in vitro replication of serum-derived hepatitis $C$ virus and virus/host cell interactions $\mathrm{J}$ Hepatol 2007; 46:1-5

[22 ] Hayward DG, Fry AM. Nek2 kinase in chromosome instability and cancer. Cancer Lett. 2006;237:155-166.

[23] CaiZ, Chehab NH, Pavletich NP. Structure and activation mechanism of the CHK2 DNA damage checkpoint kinase. Mol Cell. 2009;35:818-29

[24] Ward IM, WuX, Chen J. Threonine 68 of Chk2 is phosphorylated at sites of DNA strand breaks. J Biol Chem. 2001;276:47755-8 
[25] Janssen A, van der Burg M, Szuhai K, et al. Chromosome segregation errors as a cause of DNA damage and structural chromosome aberrations. Science. 2011;333:1895-1898

[26] Adams RR, Carmena M, Earnshaw WC. Chromosomal passengers and the (aurora) ABCs of mitosis. Trends Cell Biol 2001; 11: 49-54.

[27] Hayashi-TakanakaY, Yamagata K, Nozaki N, et al. Visualizing histone modifications in living cells: spatio-temporal dynamics of H3 phosphorylation during interphase. J Cell Biol. 2009; 187:781-790

[28] Crosio C, Fimia GM, Loury R, et al. Mitotic phosphorylation of histone H3: spatiotemporal regulation by mammalian Aurora kinases Mol Cell Biol. 2002;22:874-85

[29] Ladstein RG, Bachmann IM, Straume O, et al. Prognostic importance of the mitotic marker phospho-histone $\mathrm{H} 3$ in cutaneous nodular melanoma. J Invest Dermatol. 2012;132:1247-1252.

[30] Mazzocca A, Giusti S, Hamilton AD, et al. Growth inhibition by the farnesyltransferase inhibitor FTI-277 involves Bcl-2 expression and defective association with Raf-1 in liver cancer cell lines. Mol Pharmacol. 2003;63:159-166

[31] Manning AL, Dyson NJ. pRB, a tumor suppressor with a stabilizing presence. Trends Cell Biol. 2011;21:433-41

[32] Baker DJ, Jin F, Jeganathan KB, et al. Whole chromosome instability caused by Bub1 insufficiency drives tumorigenesis through tumor suppressor gene loss of heterozygosity. Cancer Cell. 2009;16:475-486.

[33] Weaver BA, Silk AD, Montagna C, et al. Aneuploidy acts both oncogenically and as a tumor suppressor. Cancer Cell. 2007; 11:25-36.

[34] Lin ZZ, Jeng YM, Hu FC, et al. Significance of Aurora B overexpression in hepatocellular carcinoma. Aurora B overexpression in HCC. BMC Cancer. 2010;10:461

[35] Tanaka S, Arii S, Yasen M, et al. Aurora kinase B is a predictive factor for the aggressive recurrence of hepatocellular carcinoma after curative hepatectomy. $\mathrm{Br} \mathrm{J}$ Surg. 2008; 95: 611-619

[36] Lu G, ShanT, He S, et al. Overexpression of CENP-H as a novel prognostic biomarker for human hepatocellular carcinoma progression and patient survival. Oncol Rep. 2013;30:2238-44. 
[37] Burkhart DL, Sage J. Cellular mechanisms of tumour suppression by the retinoblastoma gene. Nat Rev Cancer.2008;8:671-82

[38] Genovese C,Trani D, Caputi M, et al. Cell cycle control and beyond: emerging roles for the retinoblastoma gene family. Oncogene. 2006;25:5201-9.

[39] van Harn $T$, Foijer $F$, van Vugt $M$, et al. Loss of Rb proteins causes genomic instability in the absence of mitogenic signaling. Genes Dev. 2010;24:1377-88

[40] Takeda S, Collins ER, Kurahashi K. The FHA domain determines Drosophila Chk2/Mnk localization to key mitotic structures and is essential for early embryonic DNA damage responses. Mol Biol Cell. 2015 ;26:1811-1828.

[41] Polo SE, Jackson SP. Dynamics of DNA damage response proteins at DNA breaks: a focus on protein modifications. Genes Dev 2011;25: 409-433.

[42] Fuller BG, Lampson MA, Foley EA, et al. Midzone activation of aurora $B$ in anaphase produces an intracellular phosphorylation gradient. Nature. 2008; 453:11321136

[43] Schwarz JK, Lovly CM, Piwnica-Worms H. Regulation of the Chk2 protein kinase by oligomerization-mediated cis- and trans-phosphorylation. Mol. Cancer Res. 2003;1: 598-609.

[44] Li J, Williams BL, Haire LF, et al. Structural and functional versatility of the FHA domain in DNA-damage signaling by the tumor suppressor kinase Chk2. Mol Cell. 2002;9:1045-54. 


\section{Figure legends}

Figure 1. Immunohistochemical detection of Chk2 and phospho-Histone H2A.X(Ser139) in diethylnitrosamine-induced HCC. A) Representative image of livers of female Wistar rats used as control (left panel) and rats HCC (right panel) which were given diethylnitrosamine $50 \mathrm{mg} / \mathrm{L}$ ad libitum in their drinking water for a period of 10 weeks. Scale bar, 10mm. B) Liver sections (HCC 14 weeks) were analyzed for Chk2 and phospho-Histone H2A.X(Ser139) expression (see nuclear staining in the right panels, Scale bar $200 \mu \mathrm{m}$.). C) and D) Five animals for each time point were sacrificed on week 4, 6, 8, 10, 14. Chk2 and phospho-Histone H2A.X(Ser139) expression were determined according to $\mathrm{H}$-score method. The average positive percentage was calculated, and a $\mathrm{H}$-score between 0 and 300 was obtained where 300 was equal to $100 \%$ of tumour cells stained brown strongly. The positive cut off was considered $\geq 100$. The levels of Chk2 and phospho-Histone H2A.X(Ser139) expression are indicated as fold increase of cut-off values. ${ }^{*} P<0.001$ vs 8 weeks. Chk2 expression was evaluated in the nucleus of the HCC tissue as indicated by the brown staining.

\section{Figure 2a. Detection of Chk2 protein in human HCC by immunohistochemistry.} Paraffin-embedded tissue sections were stained with a specific antibody for Chk2 and hematoxylin and eosin. A) Normal liver with a moderate, diffuse cytoplasmic Chk2 staining. B) Chk2 immunoreactivity in cancer tissue from a tumour classified Grade 1 characterized by a weak focal cytoplasmic Chk2 staining. C) and D) Chk2 immunoreactivity in cancer tissue from a tumour classified Grade 2 with a strong Chk2 perinuclear and moderate nuclear accumulation. E) Chk2 immunoreactivity in cancer tissue from a tumour classified Grade 3 with a clear and strong nuclear staining, without cytoplasmic and perinuclear Chk2 staining. Scale bar $100 \mu \mathrm{m}$.

\section{Figure 2b. CHK2 Expression in HCC subtypes from TCGA database.}

CHK2 expression from RNA-seq data was retrieved for 101 patients with TP53 mutations and 85 patients with CTNNB1/beta-catenin mutations, using the LIHC cohort of 377 cases (accessed on November 16, 2016). Patients with both TP53 and CTNNB1 
mutations were excluded from the analysis. Expression levels are expressed as log2exp (absolute levels) or z-score (expression levels in HCC samples normalized to the expression levels in the reference population).Graph shows mean \pm SEM, ${ }^{*} p<0.0001$

Figure 3. Defective chromosome segregation causes Chk2 overexpression in hTERT immortalized human hepatocytes. A) Examples of chromosome numbers from metaphase spreads of HCT116, Huh7 and HuS0gen cells (upper panels). In the bottom panels individual chromosome numbers percentage from metaphase spreads of HCT116, Huh7 and HuSOgen cells are represented $(n=20)$. B) Representative immunoblot out of three independent experiments demonstrating the protein levels of Chk2 in indefinite cultured HCT116 and Huh7 cells, in HuSOgen cells and HuSOgen cells after 30 generations in culture (HuS30gen). C) Example of chromosomes from metaphase spreads of HuS30gen exhibiting numerical and structural (red arrow) alterations; the histogram shows individual chromosome numbers percentage in HuS30gen ( $n=20)$. D) Representative immunoblot out of three independent experiments demonstrating the protein levels of phospho-Histone H2A.X (Ser139), a hallmark of DNA damage, in indefinite cultured HCT116, Huh7, HuSOgen and HuS30gen cells.

Figure 4. Chk2 overexpression is linked to lagging chromosomes. A) Chk2 colocalizes with chromosomal structures only in HuS30gen cells. Asynchronously growing HCT116, Huh7, HuS0gen and HuS30gen cells were synchronized in metaphase by releasing cells from thymidine treatment into MG132. Metaphase mitotic spindles were monitored by immunofluorescence and typical examples are given, endogenous Chk2 (red); kinetochores, CENP-H (green); chromosomes are stained with Hoechst 33342 (blue). Chk2 co-localization with chromosome structures was quantified in metaphases (mean $\pm S D ; n \geq 300$ metaphases) B) HuSOgen that express low levels of protein Chk2 were stably transfected with HA-tagged Chk2 and asynchronously growing cells were evaluated for lagging chromosomes; typical examples of lagging chromosomes containing anaphase and telophase are given (HA-Chk2, red; CENP-H, green; chromosomes, Hoechst 33342, blue). C) Asynchronously growing HuS30gen 
cells expressing H2B-GFP (to visualize in vivo lagging chromosomes) were analyzed by live cell microscopy. Left, phase-contrast images; middle, fluorescence images; right, merge. Scale bar, $10 \mu \mathrm{m}$. D) Asynchronously growing cells, HCT116, Huh7, HuS0gen, HuS30gen, and HuSOgen stably transfected with HA-tagged Chk2 (HuS-Chk2) were stained with CENP-H (constitutive component of kinetochores) and Hoechst 33342 (chromosomes). Anaphase and telophase lagging chromosomes were counted by immunofluorescence, (mean $\pm S D ; n=500$ cells) ${ }^{*} p<0.01$ vs HuSOgen. ${ }^{* *} p<0.05$ vs HuS-mock

Figure 5. Overexpressed/phosphorylated Chk2 is correlated with increased chromosomal missegregation. A) HuS30gen cells were stably retrovirally-transduced either with a control shRNA (shCTL) or shRNAs targeting CHK2 (shChk2). Chk2depleted HuS30gen cells were used to reconstitute expression of CHK2 by introducing a shRNA resistant mutant of CHK2 (myc-Chk2resist). The protein levels were determined by immunoblotting and a representative example is shown. B) Asynchronously growing cells HuS30-shCTL, HuS30-shChk2 and Chk2-depleted HuS30gen reconstituted with shRNA resistant mutant of CHK2 were stained with CENP-H (kinetochores) and Hoechst 33342 (chromosomes). Anaphase and telophase lagging chromosomes counted by immunofluorescence, (mean \pm SD; $n=500$ cells), * $p<$ 0.01 vs controls. C) The mitotic index of asynchronously growing cells HuS30-shCTL, HuS30- shChk2 and Chk2-depleted HuS30 reconstituted with shRNA resistant mutant of $\mathrm{CHK} 2$ was measured by counting the mitotic figures (prophase, metaphase, anaphase, and telophase) positively stained for phosphorylated histone $\mathrm{H} 3(\mathrm{pHH} 3)$. The mitotic index was calculated as percentage of mitotic cells/total cells as described in Materials and Methods. Error bars show the SD from the means of three independent experiments, ${ }^{*} p<0.01$ vs controls. D) Chk2 phosphorylation levels on Thr68 and Thr387. Representative immunoblot out of three showing asynchronously growing HCT116, HuSOgen, HuS30gen, and HuSOgen stably transfected with Chk2 (HuS-Chk2). Cell lysates were immunoblotted against $p$-Thr68-Chk2 and p-Thr387-Chk2. E) Activated Chk2 localizes with mitotic structures in HuS30gen cells. Example of metaphase, anaphase and telophase from unstable HuS30gen cells and stable HCC cell line, 
Huh7. Activated Chk2 (p-Thr68-Chk2, red) co-localizes with CENP-H, constitutive component of kinetochores (green) only in HuS30gen. Arrowheads indicate lagging chromosomes, (Hoechst 33342, blue). Scale bar, $10 \mu \mathrm{m}$.

\section{Figure 6. Up-regulated Chk2 cooperates with Aurora B for chromosome condensation and cytokinesis}

A)HuSOgen cells showing expression and localization of endogenous Chk2 (red), Aurora B kinase (green), and chromosomes (Hoechst 33342, blue) B) Co-localization of Aurora B kinase (green) with HuSOgen overexpressing HA-tagged Chk2 (red) in mitoses with lagging chromosomes (Hoechst 33342, blue). Cells were fixed and analyzed by fluorescence microscopy. Arrows indicate spindle midzone region, arrowheads indicate midbody region. C) HuSOgen cells overexpressing HA-tagged Chk2 were stained with antibody anti-HA (red) and phospho-Histone $\mathrm{H} 3$ (green) to show co-localization of Chk2 with missegregating chromosomes. Scale bar, $10 \mu \mathrm{m}$.

Figure 7. FHA domain is essential for Chk2 localization and contributes to sustain lagging chromosomes. Red fluorescent protein was conjugated at the $\mathrm{N}$ terminus of each Chk2 mutant. Left, schematic diagrams. RFP was omitted. RFP-Chk2 mutants were transfected in HuS30gen cells, synchronized and blocked in metaphase by treatment with MG132 for 2 hours, the cells were analyzed by fluorescence microscopy. A) Expression and subcellular localization of RFP-Chk2. RFP-Chk2 decorates the spindle poles (two red dots), and localizes with kinetochores (CENP-H, green), chromosomes are stained with Hoechst 33342, blue. B) RFP-Chk2D347A, kinase dead mutant (KD), localizes similarly to RFP-Chk2. C) RFP-Chk2 $\triangle F H A$ does not localize to mitotic structures. D) RFP-FHA localizes to spindle poles, kinetochores. FHA domain tagged with RFP is similar to RFP-Chk2. Scale bar, $10 \mu \mathrm{m}$. E) Quantification of lagging chromosomes during anaphases and telophases in HuSOgen cells stably expressing Chk2(KD), Chk2 $\mathrm{FHA}$ and Chk2FHA mutants. Chk2 FHA mutant significantly reduces the number of lagging chromosomes as a result of the dominant 
negative effect towards endogenous Chk2. mean $\pm S D ; n=400$ anaphases and telophases. ${ }^{*} p<0.01 \mathrm{vs}$ control

Figure 8. P53 and phosphorylated Rb expression in HCT116, HuS30gen and HuS0gen. A) Representative immunoblot out of three showing the protein levels of p53 in HCT116, Huh7, HuS30gen and HuS0gen cells. In the same blot are represented p53 expression levels of HuSOgen cells after treatment with DNA-damaging agent 5fluorouracil (5-FU, 1 $\mu \mathrm{M})$. B) Representative images of HCT116, Huh7 and HuS30gen cells fixed and stained with an antibody directed against the mutated codon TGT at position 220 of p53 protein. Huh7 cells, previously characterized as p53 mutated (see reference 30), are used as positive control. C) Representative immunoblot out of three independent experiments showing the protein levels of $\mathrm{Rb}$ and phosphorylated $\mathrm{Rb}, \mathrm{p}-\mathrm{Rb}$ (Ser807/811), in HCT116, HuS30gen and HuSOgen cells. D), E) and F) Mitoses of asynchronously growing HCT116, HuS30gen and HuSOgen cells stained with the antibody specific for phosphorylated $\mathrm{Rb}$ and analyzed by fluorescence microscopy; p-Rb (Ser807/811) red; chromosomes (Hoescht 33342, blue). Scale bar, $10 \mu \mathrm{m}$.

Figure 9. A proposed schema illustrating the mechanism by which Chk2 overexpression and mislocalization sustains defective mitoses throughout hepatic tumorigenesis. Mitotic errors represented by lagging chromosomes cause DNA damage and overexpress Chk2 in genetically unstable cells. Chk2 and phosphorylated forms of Chk2 mislocalize within the mitotic structures in the presence of DNA damage contributing to increase the numbers of lagging chromosomes. 


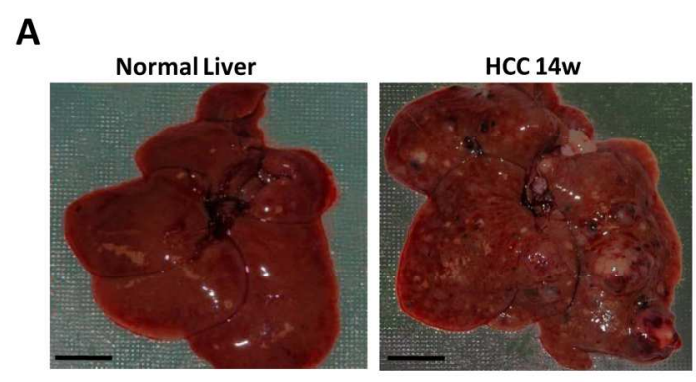

B

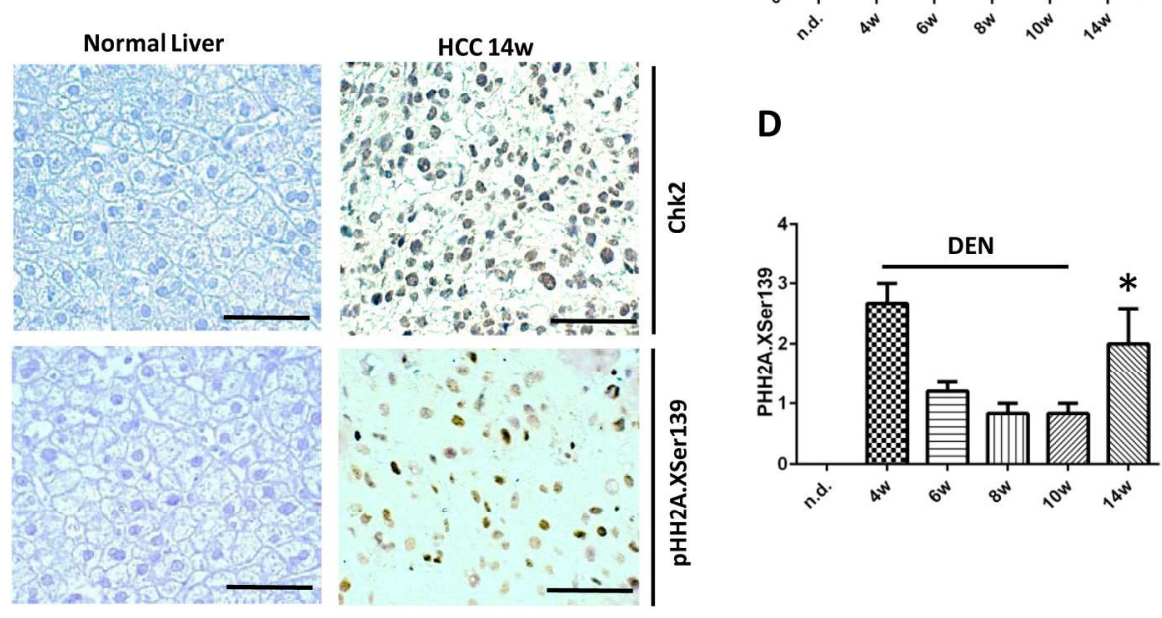

Figure 1

$181 \times 155 \mathrm{~mm}(300 \times 300$ DPI $)$ 


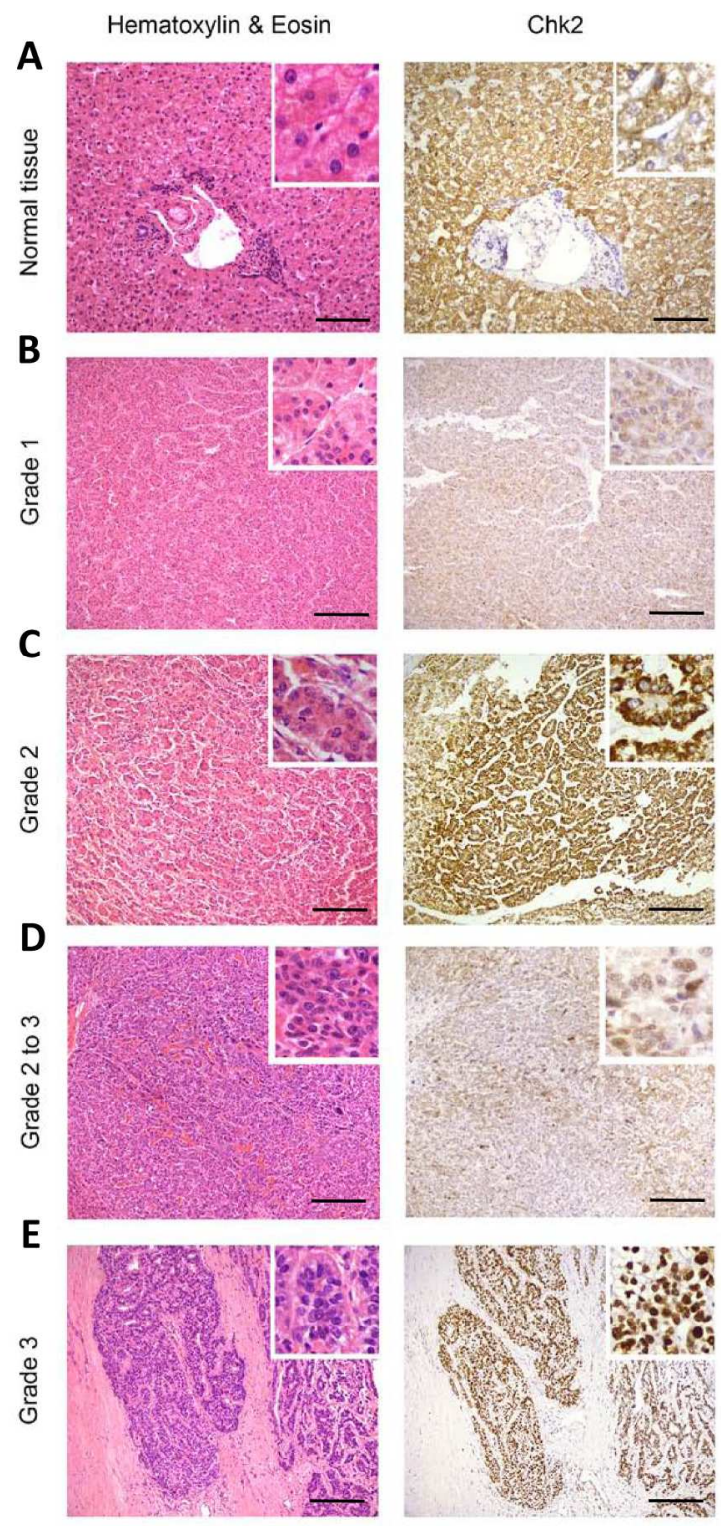

Figure $2 a$

$111 \times 227 \mathrm{~mm}(300 \times 300$ DPI $)$ 
CHK2 expression in $\mathrm{HCC}$ patients

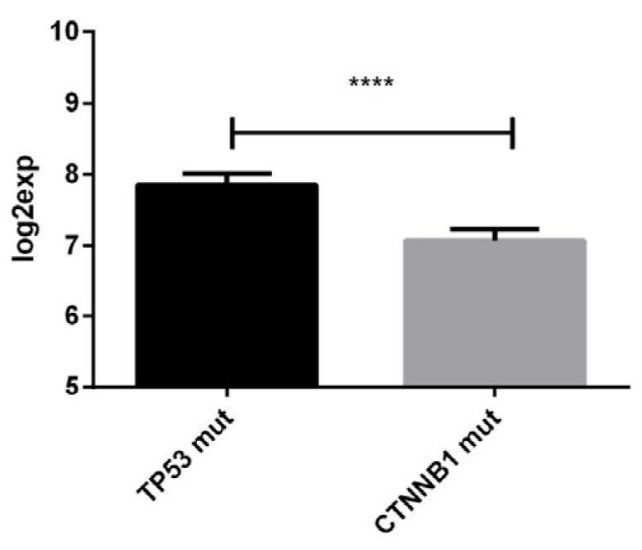

CHK2 expression in $\mathrm{HCC}$ patients

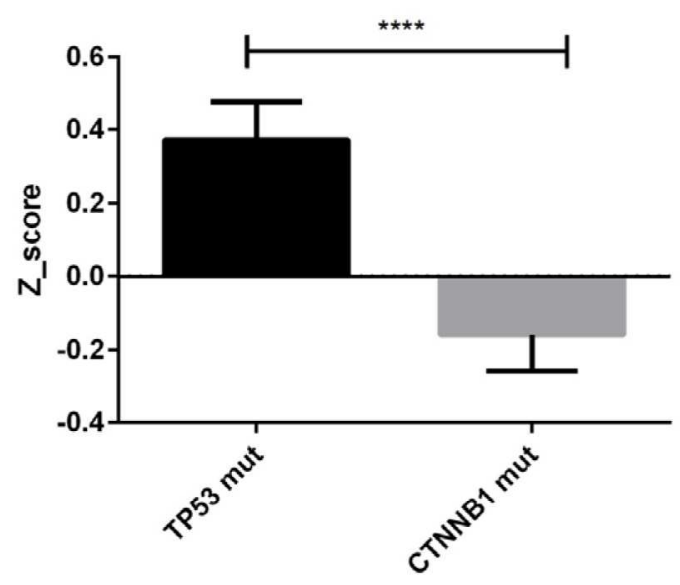

Figure $2 b$

$173 \times 192 \mathrm{~mm}(300 \times 300 \mathrm{DPI})$ 
A

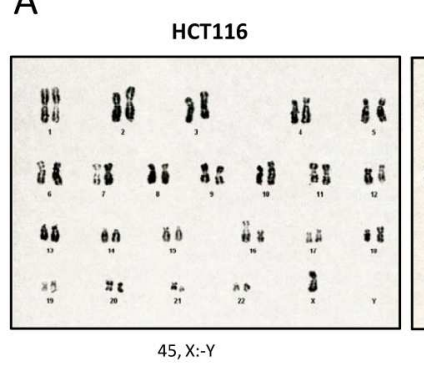

Huh7

HuSOgen
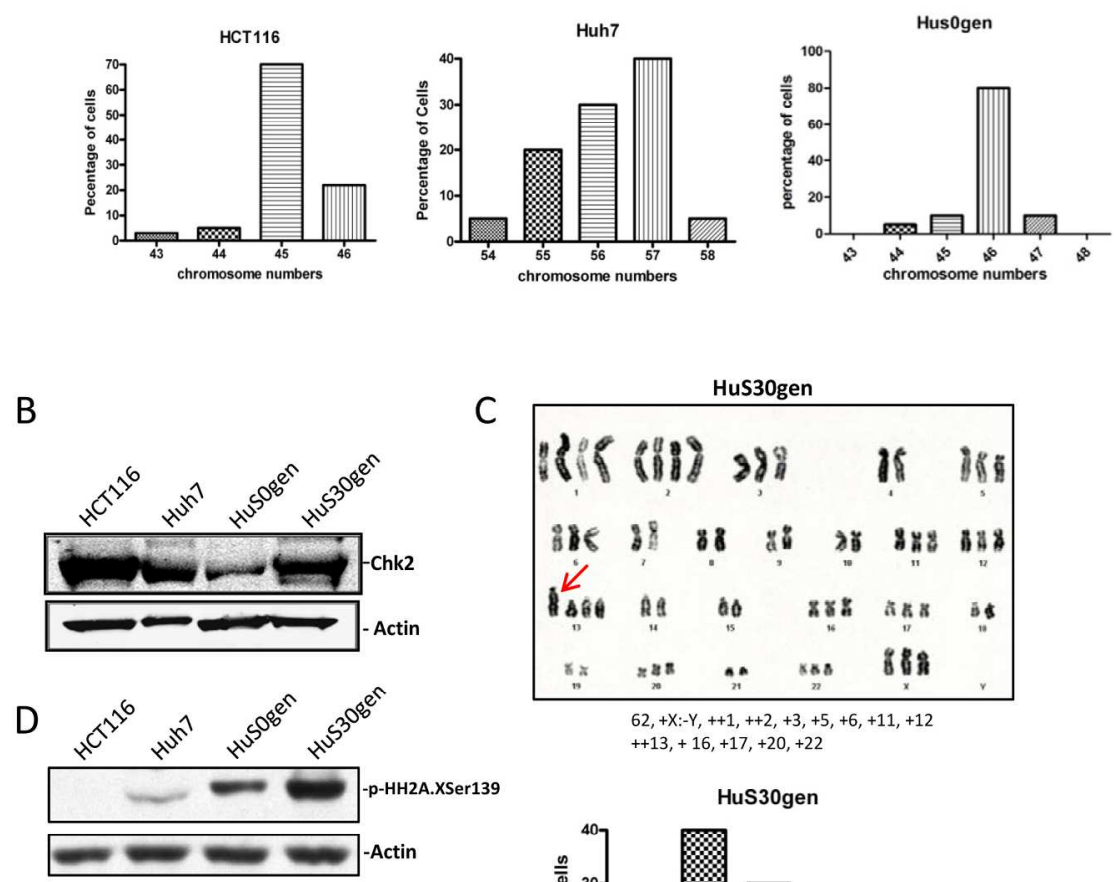

C
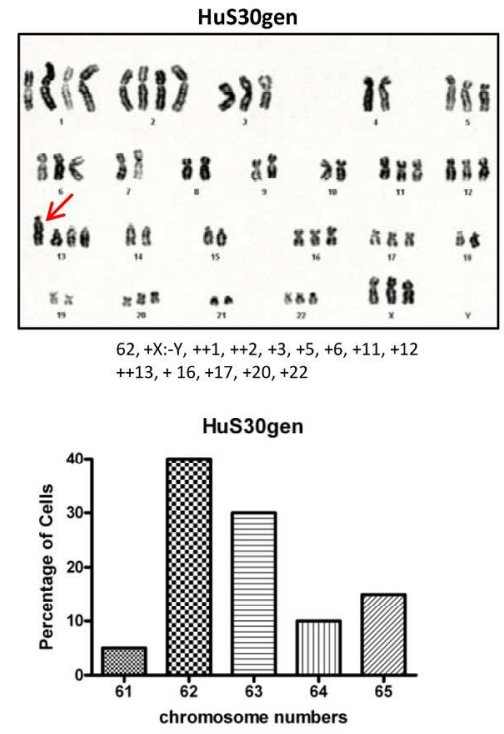

Figure 3

$186 \times 230 \mathrm{~mm}(300 \times 300$ DPI $)$ 


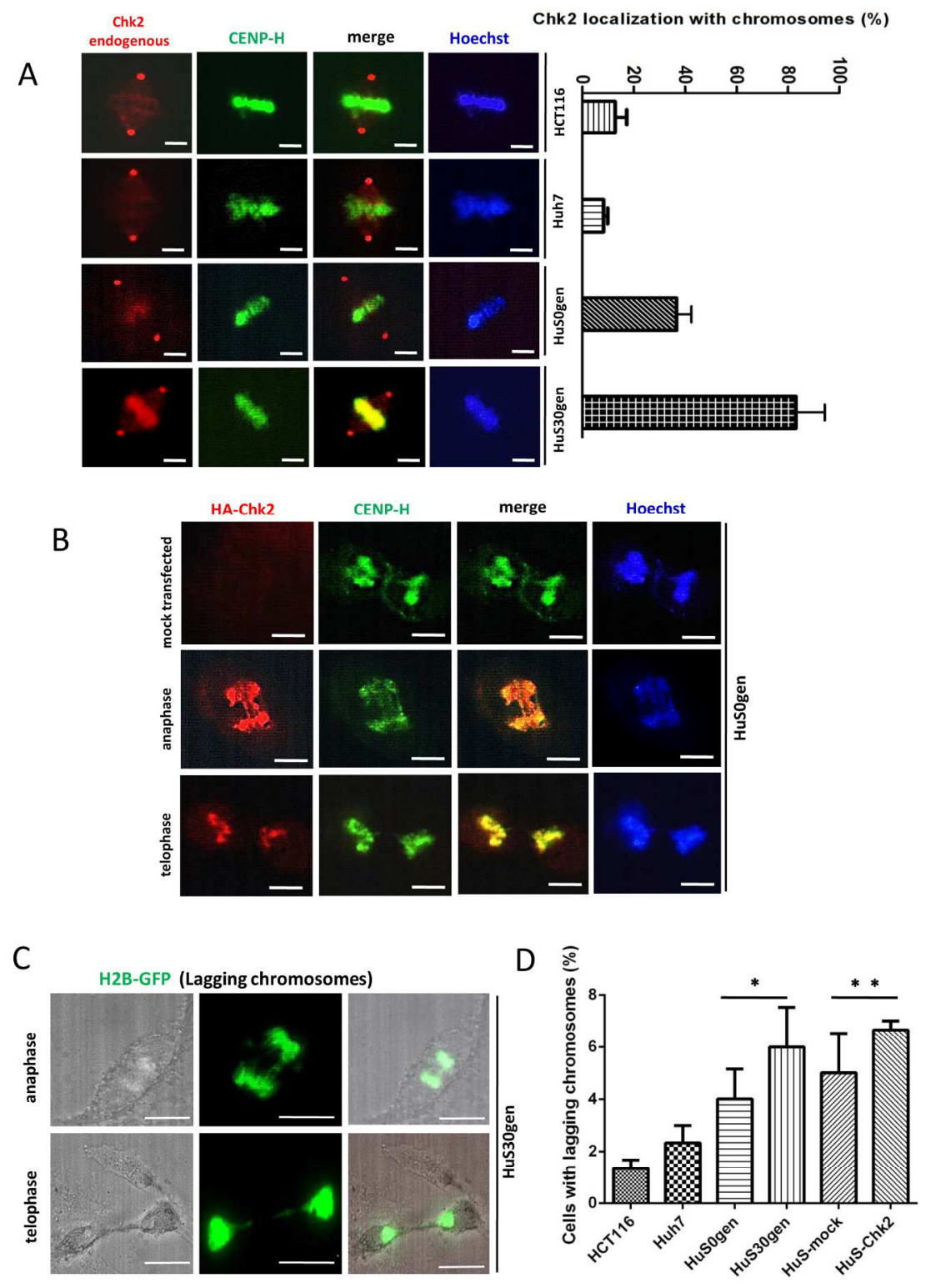

Figure 4 $169 \times 233 \mathrm{~mm}(300 \times 300$ DPI) 
A

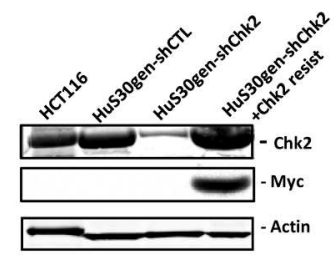

D

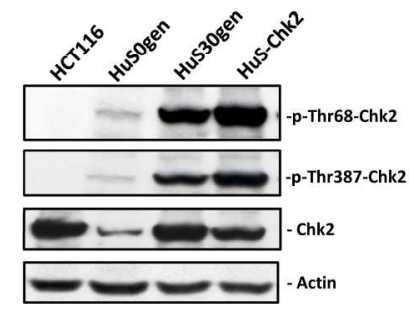

B

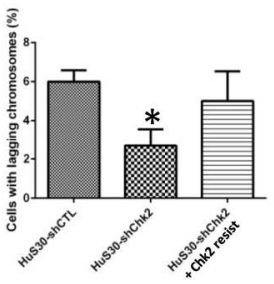

C

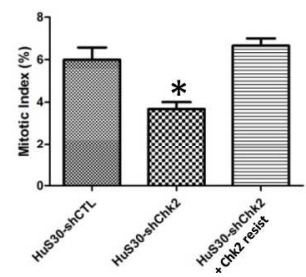

E
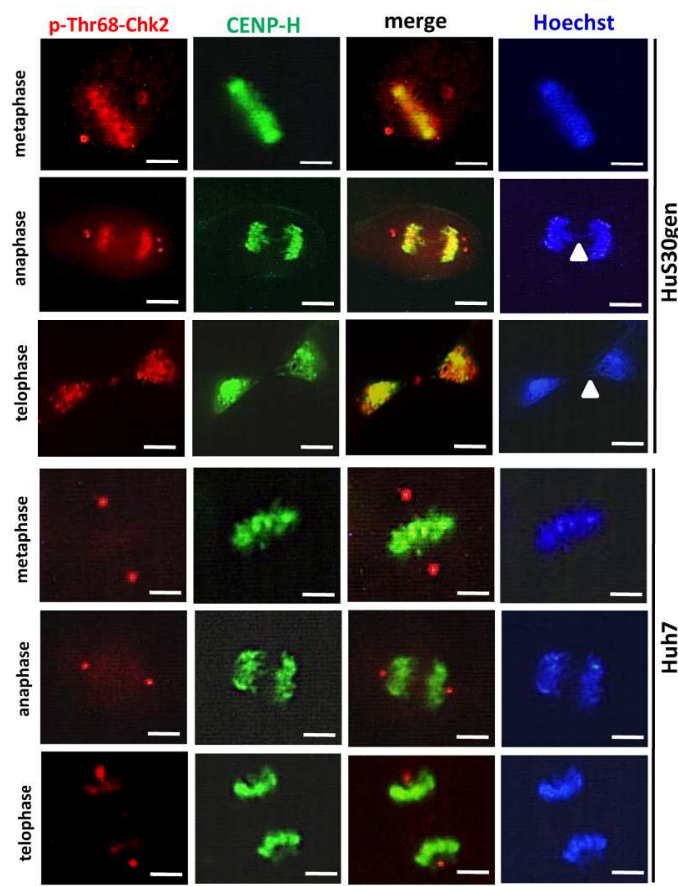

Figure 5

$158 \times 232 \mathrm{~mm}(300 \times 300$ DPI $)$ 


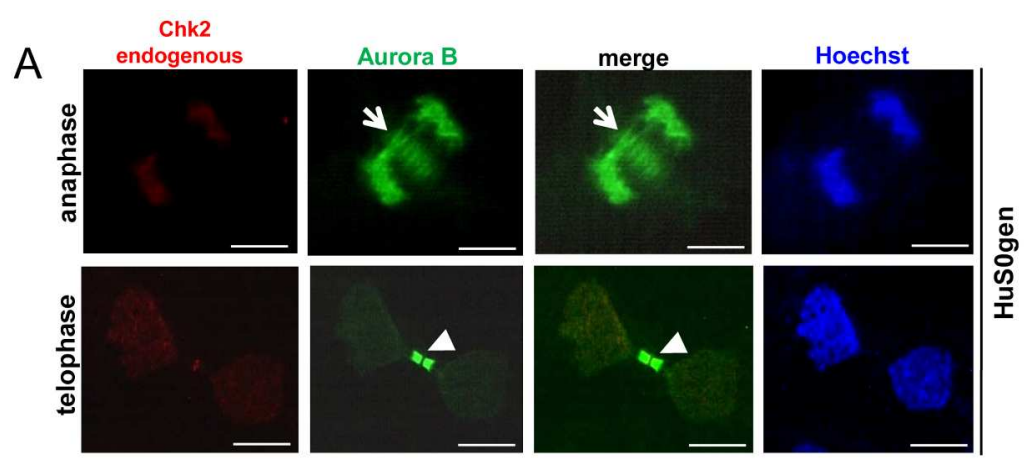

B
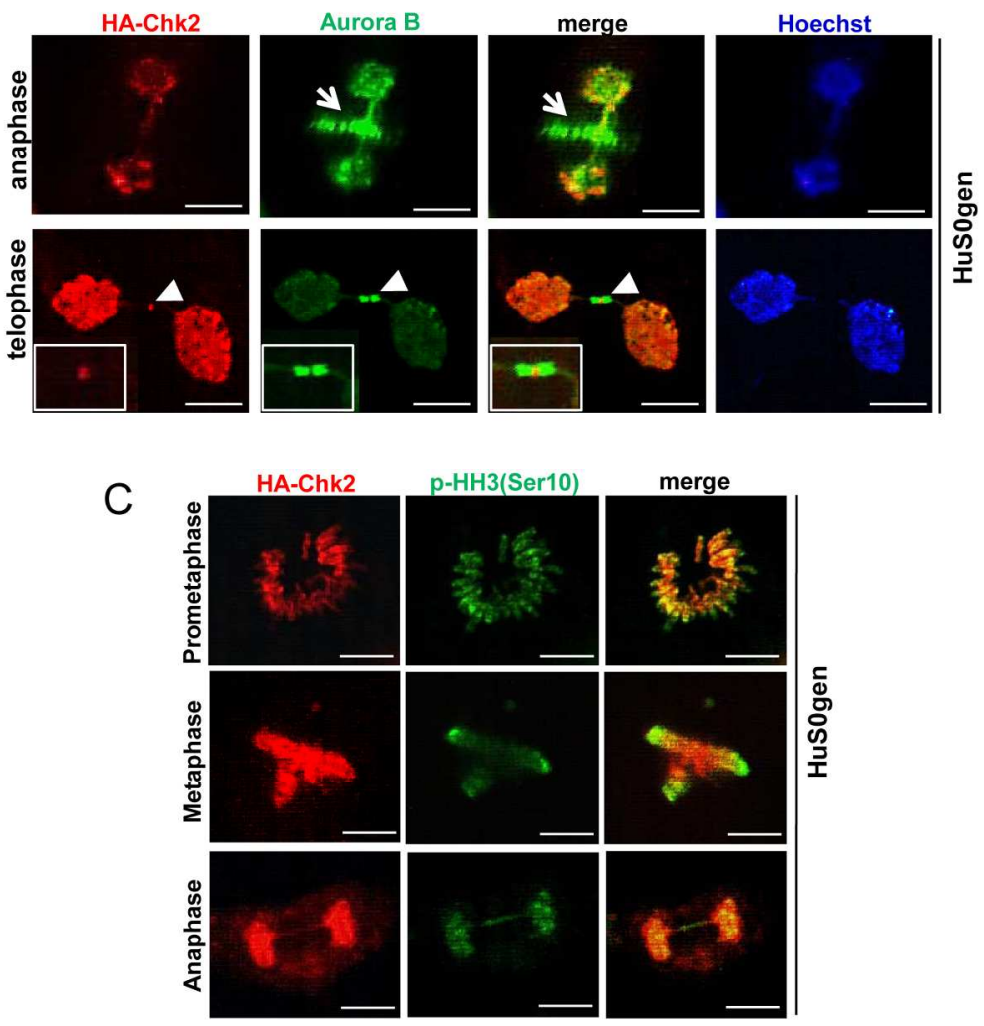

Figure 6

$161 \times 232 \mathrm{~mm}(300 \times 300$ DPI $)$ 


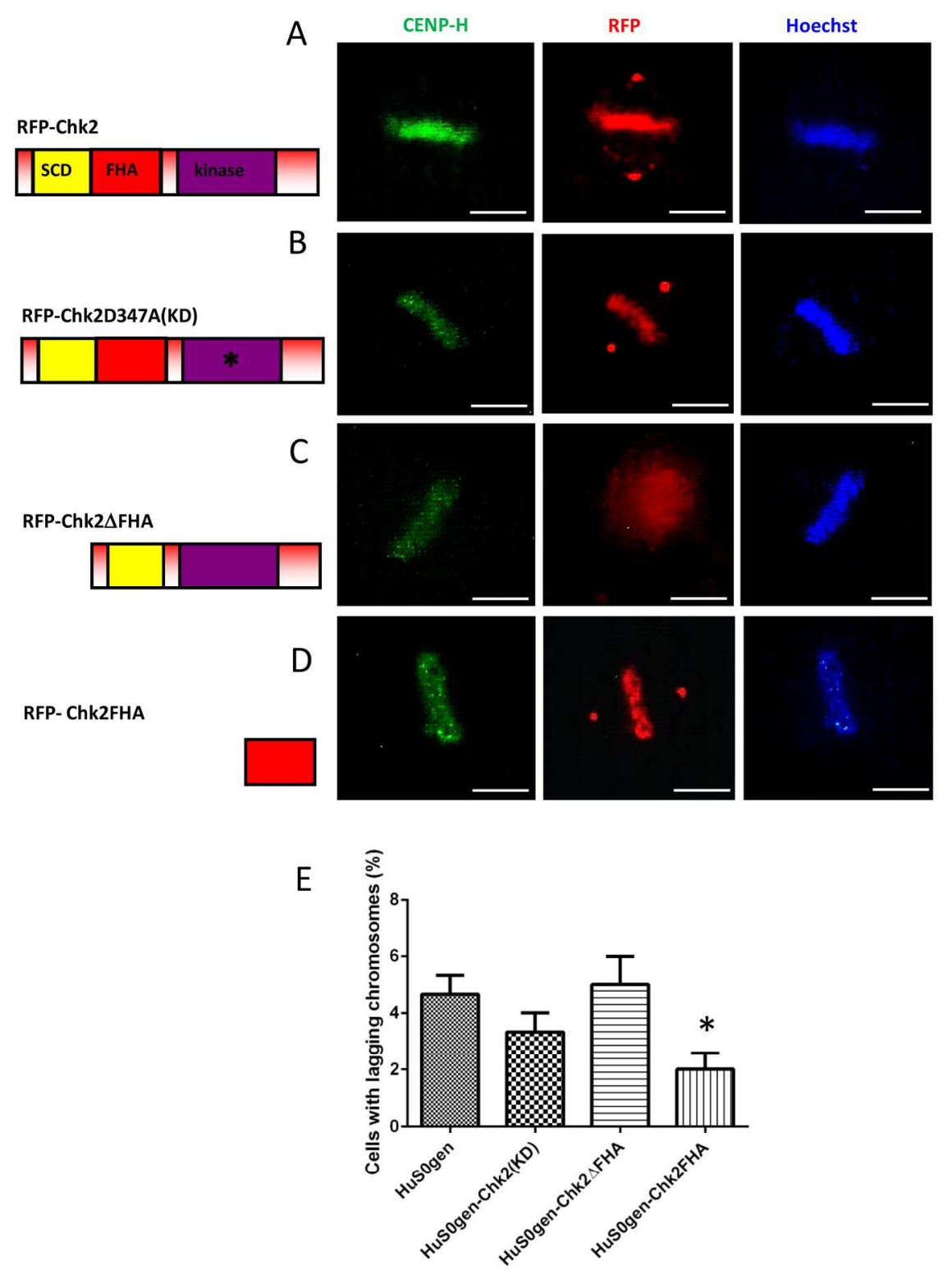

Figure 7

$170 \times 217 \mathrm{~mm}(300 \times 300 \mathrm{DPI})$ 


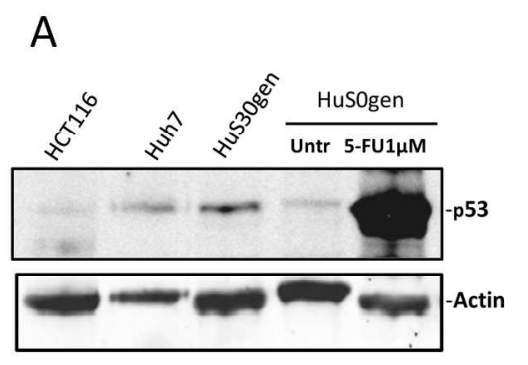

B

C

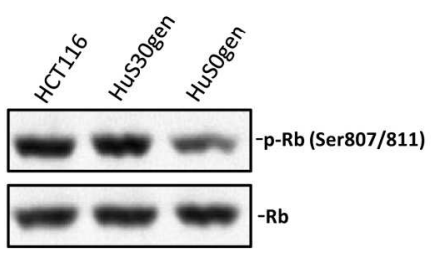

E

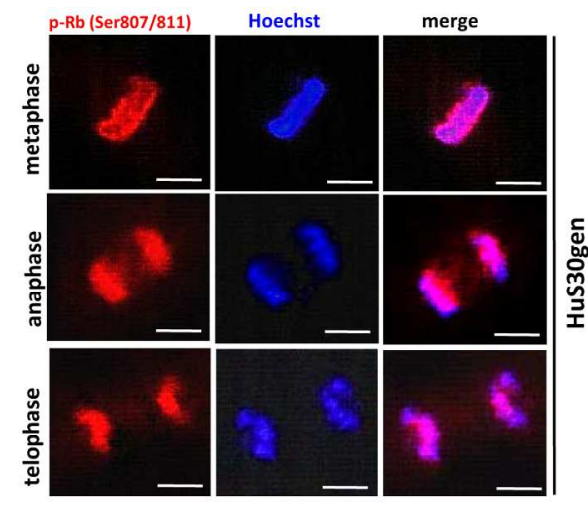

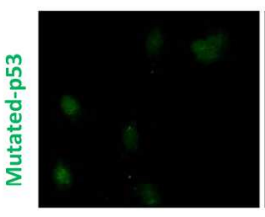

HCT116

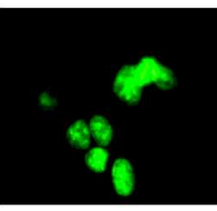

Huh7

HuS30gen

D

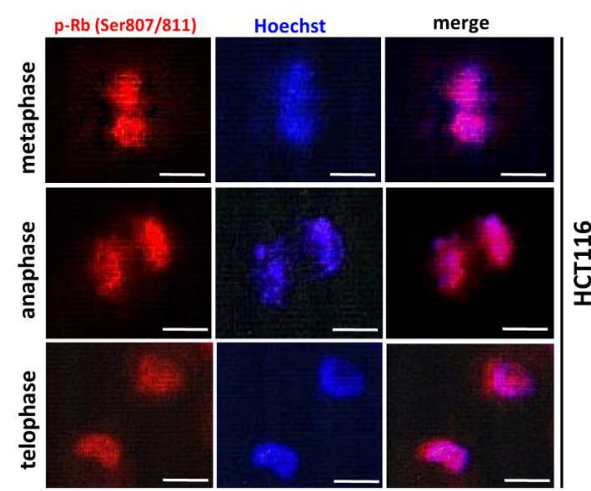

F

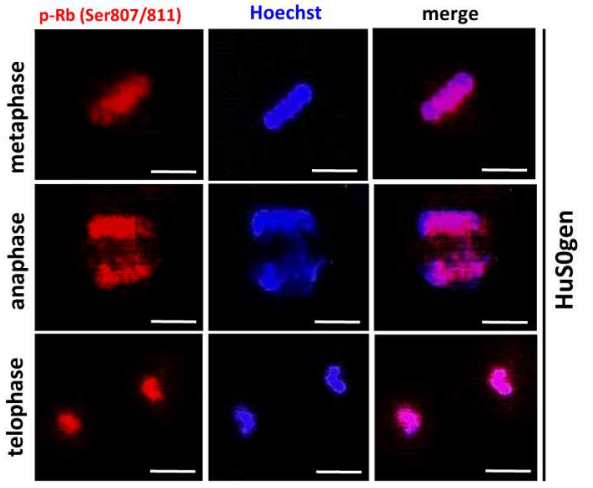

Figure 8

$168 \times 205 \mathrm{~mm}(300 \times 300$ DPI $)$ 


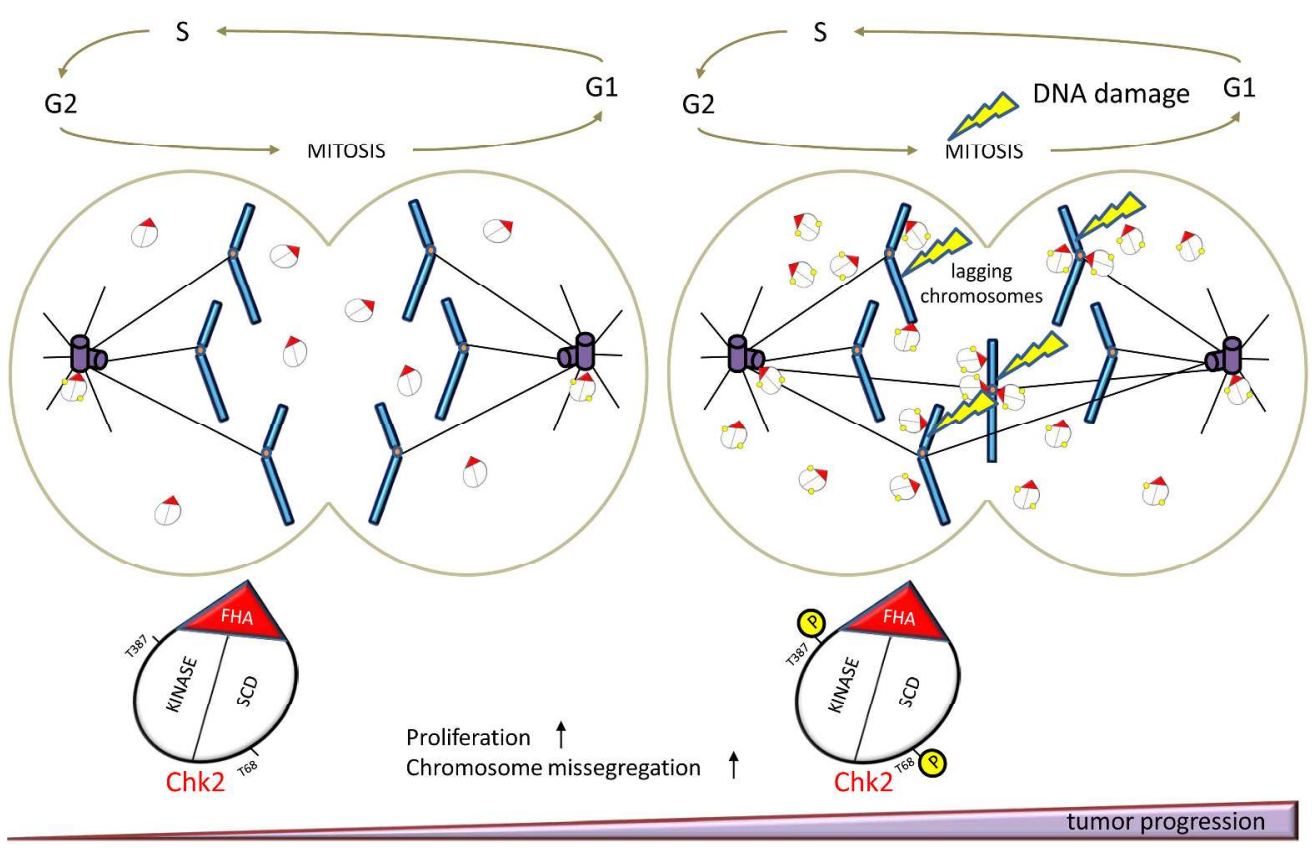

Figure 9

$276 \times 177 \mathrm{~mm}(300 \times 300 \mathrm{DPI})$ 
Table 1. Patients and Tumor Characteristics (Florence Cohort)

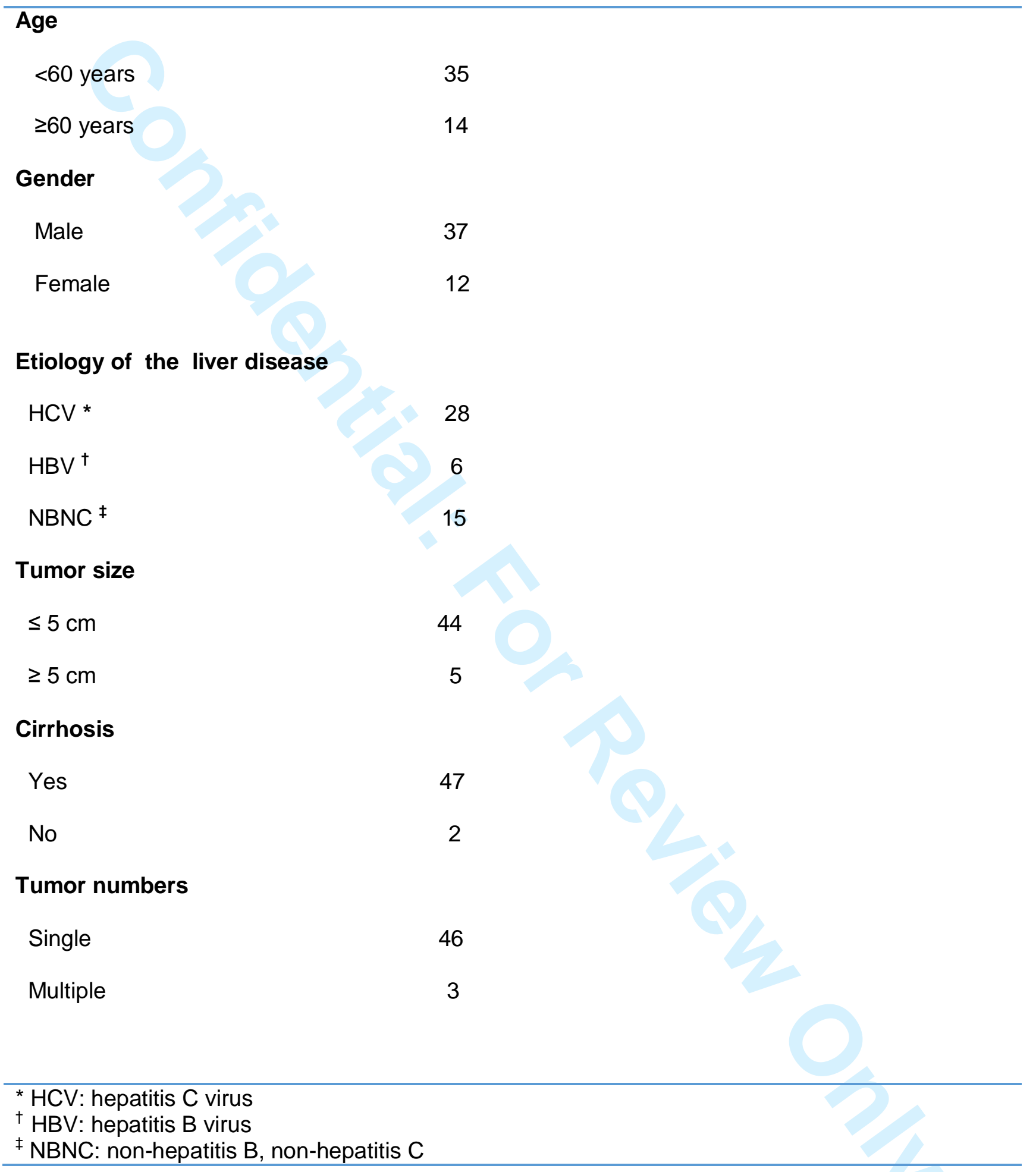


Table 2. Chk2 nuclear expression in human HCCs (Florence cohort) and its association with tumor grade. Chk2 expression was evaluated in various HCC grades and the correlation of specimens with high Chk2 was measured (Two-tailed Fisher's exact test)

\begin{tabular}{|c|c|c|c|c|}
\hline HCC grade & $\mathrm{n}$ & Chk2 ${ }^{\text {neg }}$ & Chk2 ${ }^{\text {pos }}$ & $P$ value \\
\hline II & 16 & $11(68.7 \%)$ & $5(31.3 \%)$ & $<0.001$ \\
\hline III & 33 & 7 (21.2\%) & $26(78.8 \%)$ & \\
\hline Total & 49 & $18(36.8 \%)$ & $31(63.2 \%)$ & \\
\hline
\end{tabular}


Table 3. Patients and Tumor Characteristics (Royal Free Cohort)

\section{Age}

$<60$ years

9

$\geq 60$ years

11

Gender

Male

19

Female

1

Etiology of the liver disease

$\mathrm{HCV}$ *

6

$\mathrm{HBV}^{\dagger}$

2

$\mathrm{NBNC}^{\ddagger}$

12

Tumor size

$\leq 5 \mathrm{~cm}$

15

$\geq 5 \mathrm{~cm}$

5

\section{Cirrhosis}

Yes

No

11

Tumor numbers

Single

14

Multiple

6

${ }^{*} \mathrm{HCV}$ : hepatitis $\mathrm{C}$ virus

${ }^{\dagger} \mathrm{HBV}$ : hepatitis B virus

${ }^{\ddagger}$ NBNC: non-hepatitis B, non-hepatitis C 
Table 4. Chk2 nuclear expression in human HCCs (London cohort) and its association with tumor grade. Chk2 expression was evaluated in various HCC grades and the correlation of specimens with high Chk2 was measured (Two-tailed Fisher's exact test)

\begin{tabular}{|ccccc|}
\hline \hline HCC grade & $\mathrm{n}$ & $\mathrm{Chk2}^{\text {neg }}$ & $\mathrm{Chk2}^{\text {pos }} \quad$ P value \\
\hline I & 3 & 3 & 0 & \\
II & 12 & 8 & 4 & $<0.05$ \\
III & 5 & 0 & 5 & \\
& & & & \\
\hline Total & 20 & & 9 & \\
\hline
\end{tabular}




\section{SUPPLEMENTARY METHODS}

Cell cultures and antibodies. HCT116, Huh7 were from ATCC. Human hepatocytes immortalized with TERT gene (HuS) were provided by Makoto Hijikata (Laboratory of Human Tumor Viruses, Institute of Virus Research, Kyoto University, Japan). HCT116 and Huh7 were grown in DMEM medium supplemented with $10 \%$ fetal bovine serum, $1 \%$ penicillin/streptomycin and $1 \%$ glutamine at $37^{\circ} \mathrm{C}$ and $95 \%$ humidity and $5 \% \mathrm{CO}_{2}$. HuS cells were cultured in DMEM medium supplemented with $5 \%$ fetal bovine serum, $5 \%$ normal human serum from male type $A B$ serum (Sigma), $1 \% D M S O, 0.1 \mu \mathrm{L} / \mathrm{mL}$ insulin, $0.1 \mu \mathrm{L} / \mathrm{mL}$ hydrocortisone and $2 \mathrm{ng} / \mathrm{mL}$ EGF (Calbiochem). Antibodies employed were antiChk2 (Cell Signaling, \#3440), anti-phospho-Thr68-Chk2 (Cell Signaling, \#2661), antiphospho-Thr387-Chk2 (Cell Signaling, \#2668), anti-Nek2 (Santa Cruz Biotechnology, sc\#55601), anti-y-tubulin (Santa Cruz Biotechnology, sc\#7396-R), anti-CENP-H (Santa Cruz Biotechnology, sc\#22792), anti-phospho-Histone H3(Ser10) (Cell Signaling, \#9701), anti-phospho-Rb(Ser807/811) (Cell Signaling, \#8516), anti-p53 (Santa Cruz Biotechnology, Clone Pab240, sc\#99), anti-Aurora B kinase (Sigma,\#A5102), anti- $\beta$-actin (clone AC-15) (Sigma-Aldrich). Secondary antibodies conjugated to AlexaFluor 488 and 594 (Molecular Probes) were used in immunofluorescence analysis. Secondary antibodies used in immunoblot analysis were, IRDye 800CW anti-rabbit (\#926-32210, Lycor Bioscience, Lincoln, NE) and IRDye 800CW anti-mouse (\#926-32211, Lycor Bioscience). DNA was stained with Hoechst 33342 (ThermoScientific).

Karyotype analysis. Cells were plated on Slideflask $50-70 \%$ confluent. For cell-cycle synchronization, cells were blocked twice with $2 \mathrm{mM}$ thymidine (Sigma) and released into the medium. For synchronization at metaphase, cells were released from thymidine for 6 hours, followed by treatment with MG132 (20 $\mu \mathrm{M}$; Calbiochem) for an additional $2 \mathrm{~h}$. The culture medium was removed and a hypotonic solution $(0,56 \%$ potassium chloride, $0,50 \%$ sodium citrate tribasic dehydrate) was added and incubated for 10 mins at room temperature (RT). The hypotonic solution was removed and a pre-fix solution (acetic acid $0,5 \%$ ) for 10 mins at RT was added. Next, a fixative solution (acetic acid/ ethanol) was added for 10 mins. The metaphase spread chromosomes were stained with Giemsa for Gbanding and analyzed with Zeiss Axioplan 2 microscope. 


\section{Western blot analysis}

Standard procedures were used. Briefly, protein lysates were quantified by the Bradford method (Bio-Rad, Hercules, CA) and fifty micrograms of total protein were denaturated and separated on MiniProtran TGX precast gels (Bio-Rad). After separation, the proteins were electroblotted to nitrocellulose membranes with semidry Trans-Blot (Bio-Rad) and successively membranes were incubated in a blocking solution consisting of phosphate buffered saline (PBS)/Odyssey Blocking Buffer 1:1 (PBS/OBB) (Lycor Bioscience) for 1 hour at room temperature. Membranes were then incubated overnight at $4^{\circ} \mathrm{C}$ with the appropriate antibody, washed four times with PBS-Tween $0.1 \%$ solution, and probed with the secondary IRDye antibodies according to the manufacturer's instructions. The protein bands were analysed by the Odyssey Infrared Imaging System (Lycor Bioscience) using software for protein quantification.

Retroviral infections. The following shRNAs (OriGene Technologies) were stably delivered into Hus cells by retroviral infection according to manufacturer's instructions.TF320655 is a set of plasmids containing four shRNA constructs in retroviral vector (pRFP-C-RS) to knockdown human CHK2. TR30015 is a non-effective 29mer scrambled shRNA used as control. Retroviral particles were generated by transient transfection of the Phoenixamphotropic packaging cell line as previously described. Phoenix cells plated onto 100mm dishes were transfected at approximately $80 \%$ confluence with $20 \mu \mathrm{g}$ of plasmid using Lipofectamine 2000 transfection reagent. After 48 hours, virus-containing supernatant was recovered and centrifuged, filtered $(0.45 \mu \mathrm{m}$ filters) and used immediately or aliquoted and frozen at $-80^{\circ} \mathrm{C}$. Target cells were infected with virus-containing supernatant in the presence of polybrene $(8 \mu \mathrm{g} / \mathrm{ml}$, Sigma). Retrovirally infected Hus cells were selected in puromycincontaining medium and, after 2 weeks, reduced expression of Chk2 protein was assessed by immunoblotting. A shRNA resistant mutant of $\mathrm{CHK} 2$ was generated using the mutagenesis kit (QuikChange; Agilent Technologies) yielding an exchange of four bases within the shRNA targeting sequence (5'-GCTCCTTAGAGACAGTGTCCACTCAGGAA-3') without changing the resulting amino acid sequence and cloned into the pcDNA3.1 plasmid.

Expression of Chk2 mutants. The plasmid pBabe-HA tagged Chk2 (Addgene) coding for full length human $\mathrm{CHK} 2$ was used as template to clone Chk2 into pTagRFP-C1 (Evrogen) plasmid by PCR and the construction of a kinase dead mutant (D347A) by site-directed mutagenesis primer sense 
5'-AACGGTATTATACACCGTGCATTAAAGCCAGA-3'. To generate plasmid RFPChk2 $\triangle \mathrm{FHA}$ coding a Chk2 protein lacking the FHA domain, a fragment containing amino acids 1-112 of Chk2 was isolated by PCR from pBabe-HA tagged Chk2 and cloned as a Xhol-EcoRI fragment into the pTagRFP-C1 vector. A second fragment containing amino acids 201-342 isolated by PCR was cloned in frame using Pstl-Kpnl into the vector pTagRFP-C1 harbouring the fragment 1-112. To produce RFP-Chk2FHA plasmid the Xhol and BamHI-flanked fragment containing the FHA domain was cloned from pBabe-HA tagged Chk2 into the pTagRFP-C1vector.

Immunofluorescence and live-cell microscopy. Cells were fixed in ice-cold methanol for 5 min at $-20^{\circ} \mathrm{C}$, washed twice with PBS at RT, blocked 60 minutes in $3 \%$ BSA with $0.05 \%$ NP-40 and immunostained. HuS cells were transfected with H2B-GFP (Addgene) and seeded onto Petri dishes with a $35-\mathrm{mm}$ glass bottom (Ibidi). Phase-contrast and fluorescence images were taken by using an inverted fluorescence microscope (DMIRE2; Leica) and a 40X Plan Neo 0.6 NA Ph2 objective. Live imaging was performed in air, at $37^{\circ} \mathrm{C}$ in $5 \% \mathrm{CO} 2$, using a camera (DFC300FX; Leica) and IM50 acquisition software (Leica).

Mitotic index. Cells were fixed in ice-cold methanol for $5 \mathrm{~min}$ at $-20^{\circ} \mathrm{C}$, washed twice with PBS at RT, blocked 60 minutes in 3\% BSA with $0.05 \%$ NP-40, stained with antibody against pHH3 (Ser10) and Hoechst 33342 for DNA. Ten high-power fields were examined by fluorescence microscopy, mitotic figures (profase, metaphase, anaphase, and telophase) were counted and mitotic index was calculated as percentage of mitotic green cells(pHH3)/total blue cells (Hoechst).

Statistical analysis. The significance of correlations between CHK2 expression patterns in human tissue and grade was measured by applying two-sided Fisher's exact test. All quantifications of mitotic indexes and lagging chromosomes were based on at least 3 independent experiments, in which at least 500 mitotic figures were evaluated. The data are expressed as means $\pm S D$. Statistical analysis was performed by two-tailed Student's $t$ test. 
Ethical Approval for Use of Human Material and Animal Experimentation. Informed written consent was obtained from all patients in accordance with the guidelines of University Hospital of Florence and Royal Free Hospital, London. All animal experiments were approved and performed in accordance with the regulations set forth by the Italian Ministry of Health. 


\section{SUPPLEMENTARY LEGENDS}

Figure S1. H-score examples (range 0 to 300). Case (A) weak Chk2 expression; $\mathrm{H}$ score 20. Case (B) moderate Chk2 positive; H-score 150. Case (C) remarkable Chk2 expression; H-score 230. Case (D) strong Chk2 expression; H-score 300. Scale bar, 100 $\mu \mathrm{m}$.

Figure S2. Detection of Chk2 protein in different histotype of human HCC. A) Normal liver negative control (sections treated with nonimmune antibody), B) Normal liver showing a faint cytoplasmic staining of Chk2 without nuclear detection, C) HCC pseudo-glandular histotype negative control (sections treated with nonimmune antibody), D) HCC pseudoglandular histotype with nuclear expression of Chk2, E) HCC trabecular histotype negative control, F) HCC trabecular histotype showing strong nuclear Chk2 expression, G) HCC scirrhous histotype negative control, H) HCC scirrhous histotype with the scattered cancer cells showing nuclear staining for Chk2. Scale bar, $100 \mu \mathrm{m}$.

Figure S3. A) Vascular invasion of a cluster of HCC cells showing Chk2 nuclear staining (100x magnification). B) inset shows the presence of a cell with lagging chromosomes (250x magnification).

Figure S4. G-banding images of chromosome spreads and Chk2 localization through mitotic phases in HuS30gen cells. A) The chromosomes of metaphase spread of HuS30gen exhibit numerical and structural (red arrows) abnormalities. B) HuS30gen cells were synchronized by thymidine treatment and then released into medium. Bipolar spindle assembly and chromosome alignment were monitored by immunofluorescence and typical examples are given, endogenous Chk2 (green), Ytubulin (red), chromosomes are stained with Hoechst 33342 (blue).

Figure S5. Nek2 interactions with mitotic structures in HCT116, Huh7, HuSOgen and HuS30gen. A) Representative immunoblot out of three independent experiments showing the Nek2 protein expression in indefinite cultured HCT116 and Huh7 cells, or in HuSOgen and HuS30gen cells, ${ }^{\star} p \leq 0.001$ vs HCT116 or Huh7. B) Nek2 localization in metaphase blocked HCT116 and Huh7 cells. Nek2 (arrows) localizes with poles of mitotic spindle in both cell lines (Nek2, red; Y-tubulin, green; chromosomes, bleu). C) HuSogen and 
HuS30gen were synchronized and blocked in metaphase. Representative image of Nek2 (arrow) localization in HuSOgen and HuS30gen (Nek2, green; Y-tubulin, red; chromosomes, bleu). Both cell lines show low expression levels of Nek2 protein. Scale bar, $10 \mu \mathrm{m}$. 

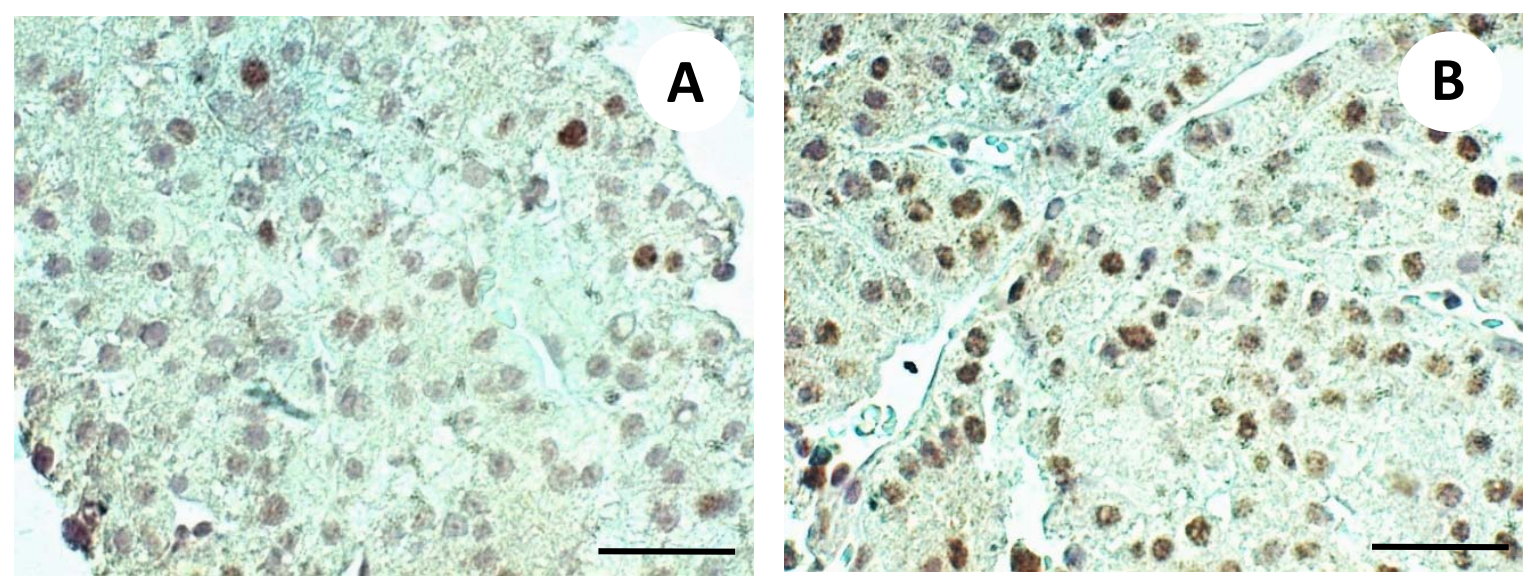

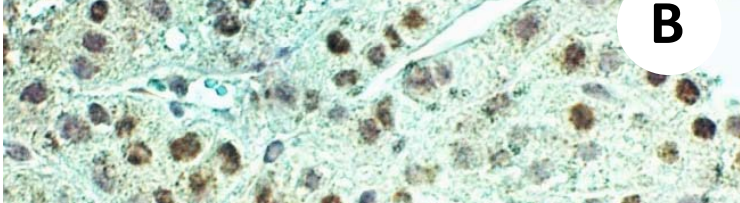

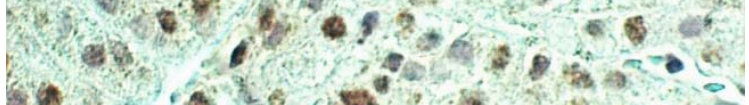

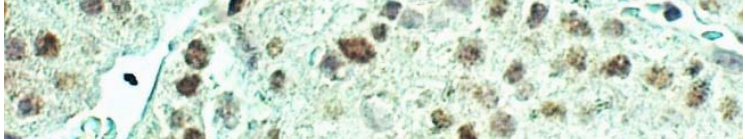

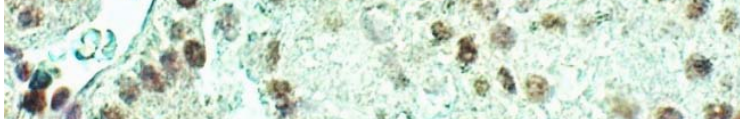
20.

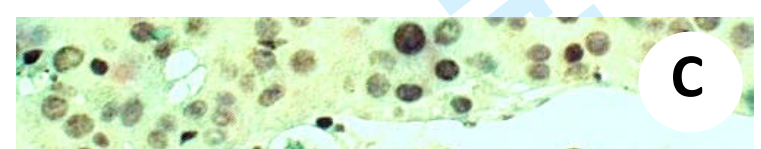

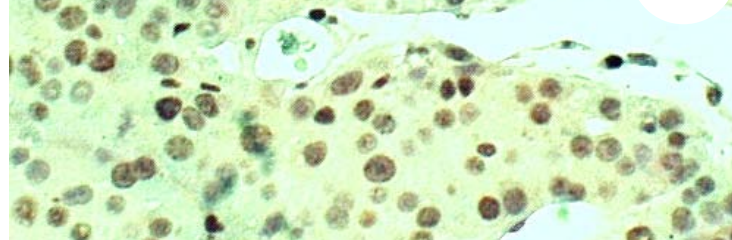
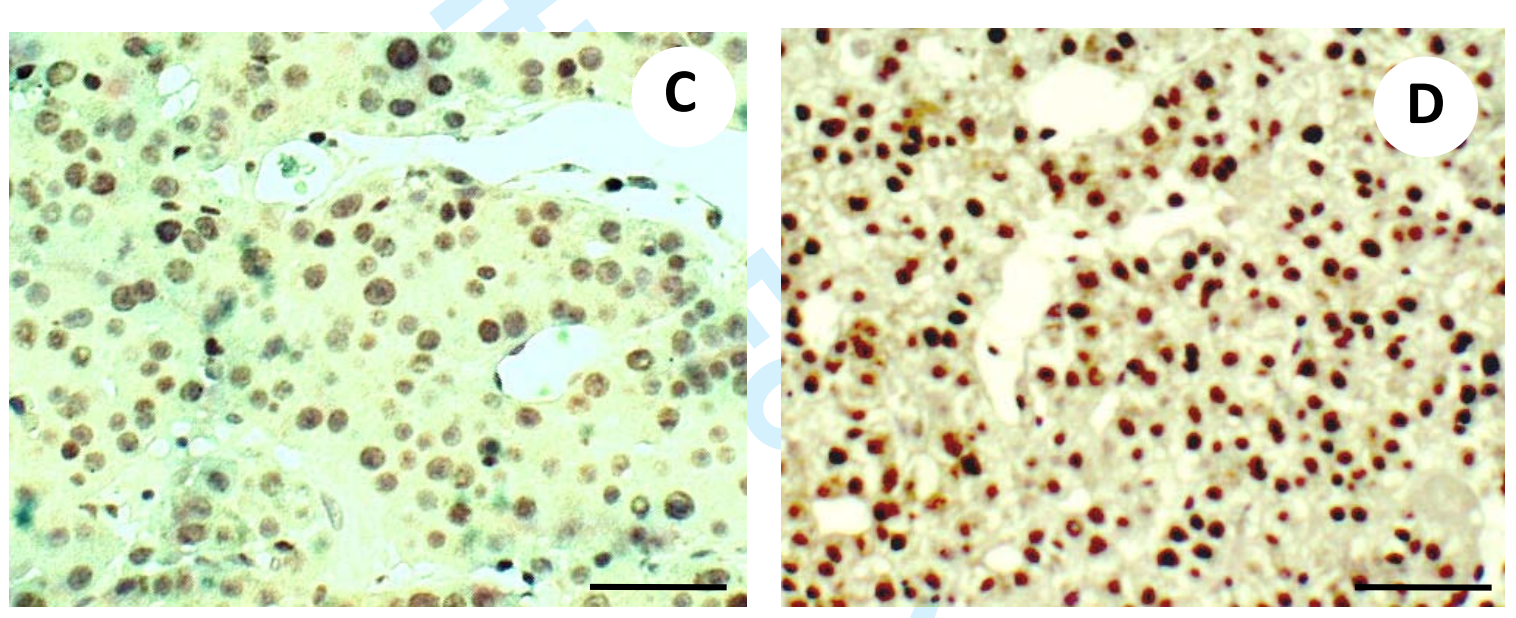

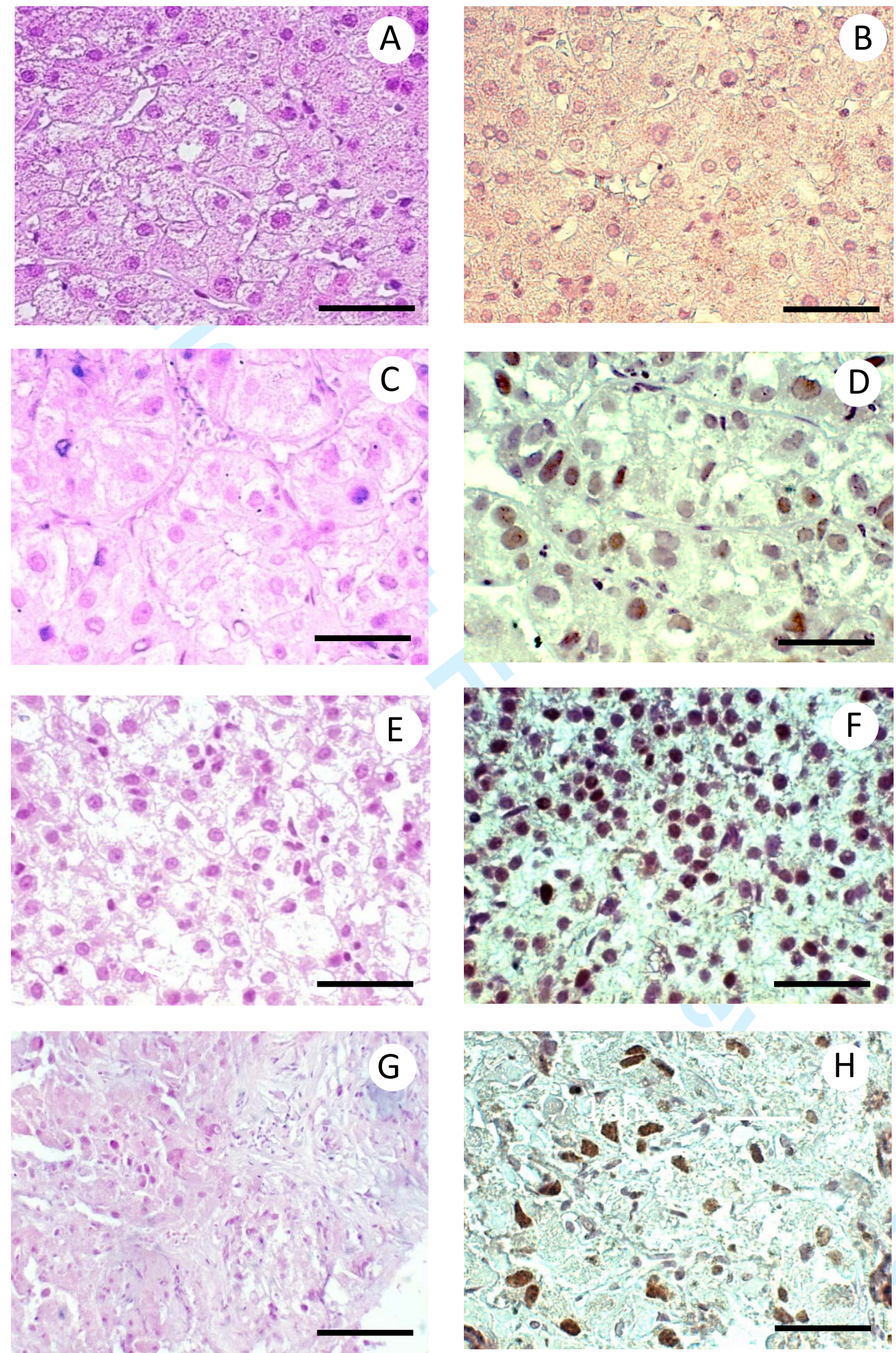
A
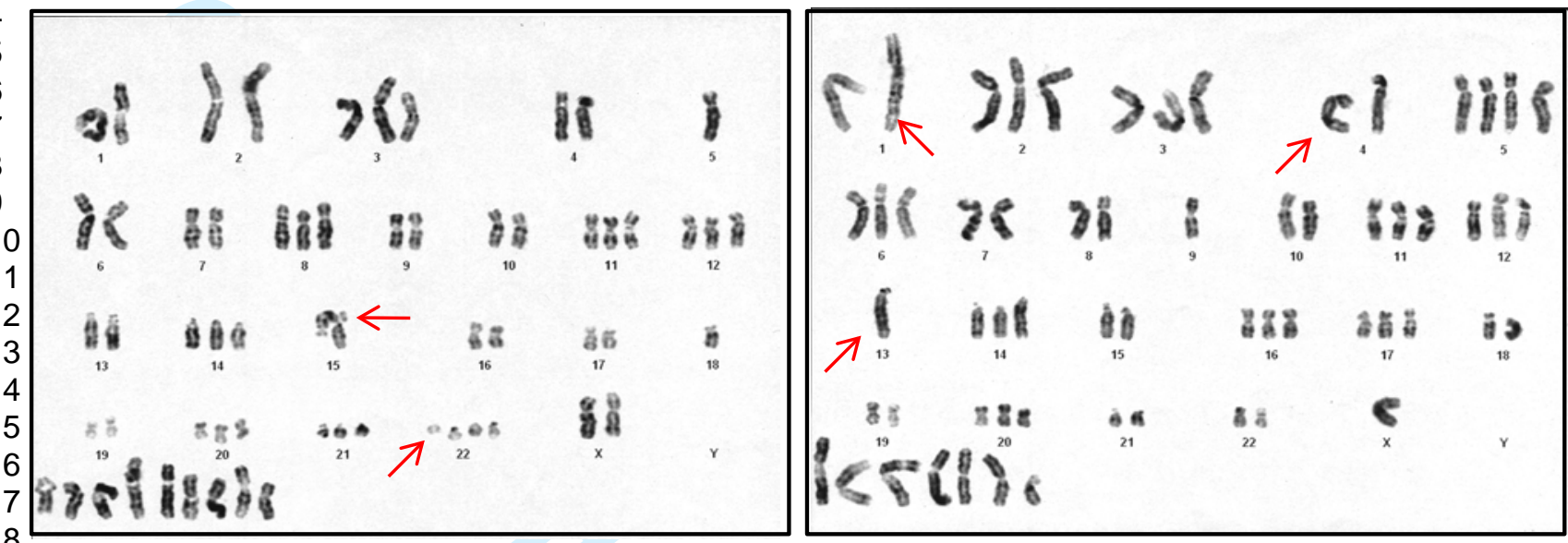

19

20

21

22

23

24

25

26

27

28

29

30

31

32

33

34

35

36

37

38

39

40

41

42

43

44

45

46

47

48

49

50

51

52

53

54

55

56

B

Chk2
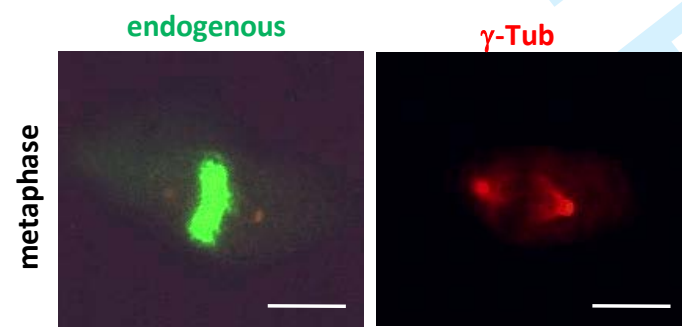

Hoechst
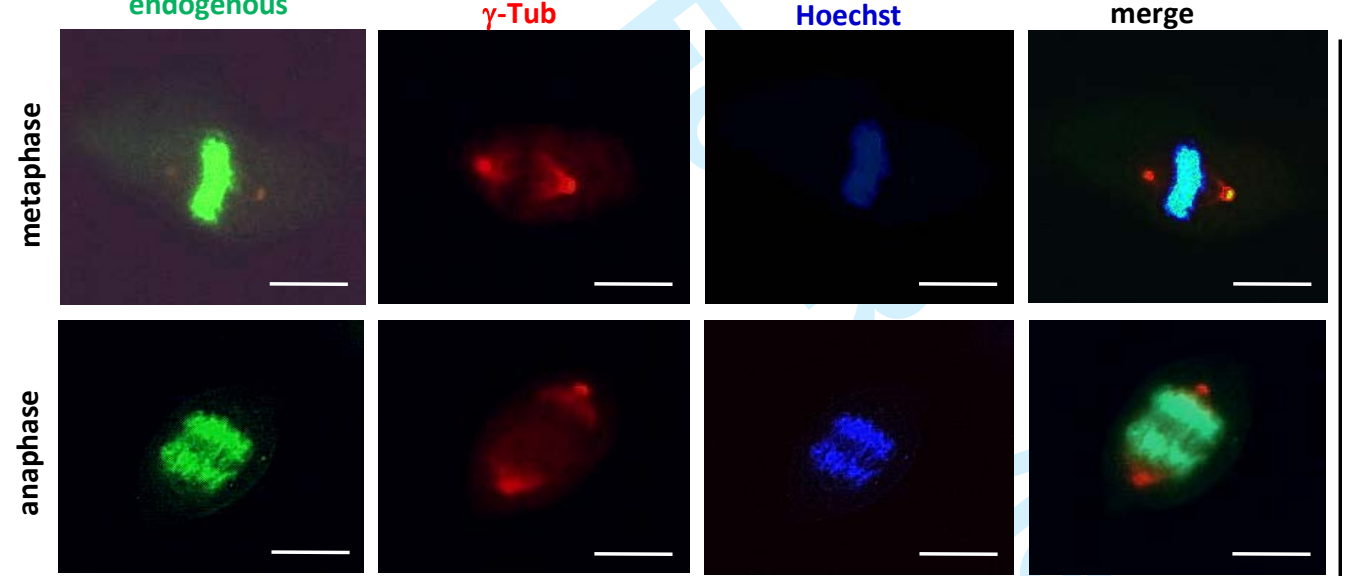

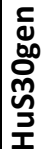
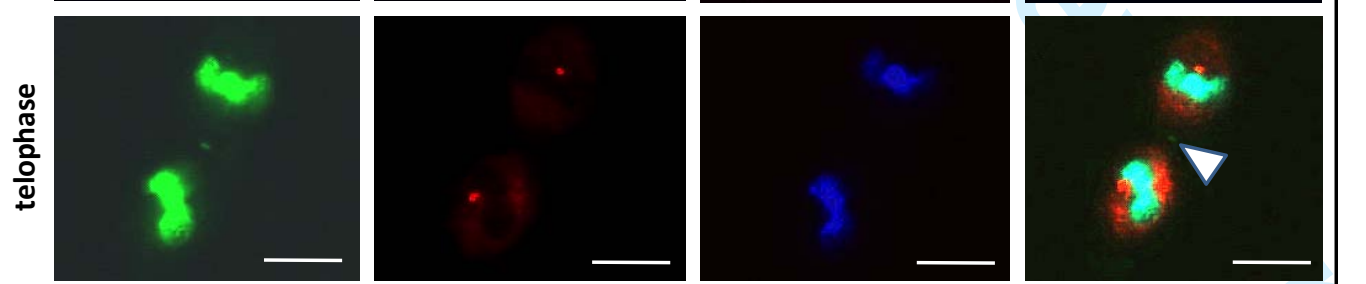

https://mc.manuscriptcentral.com/gut

57 


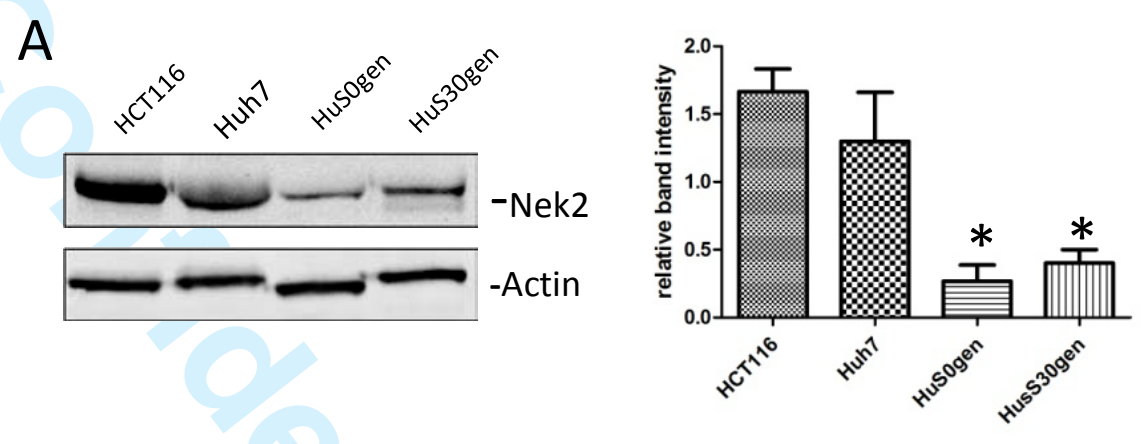

B

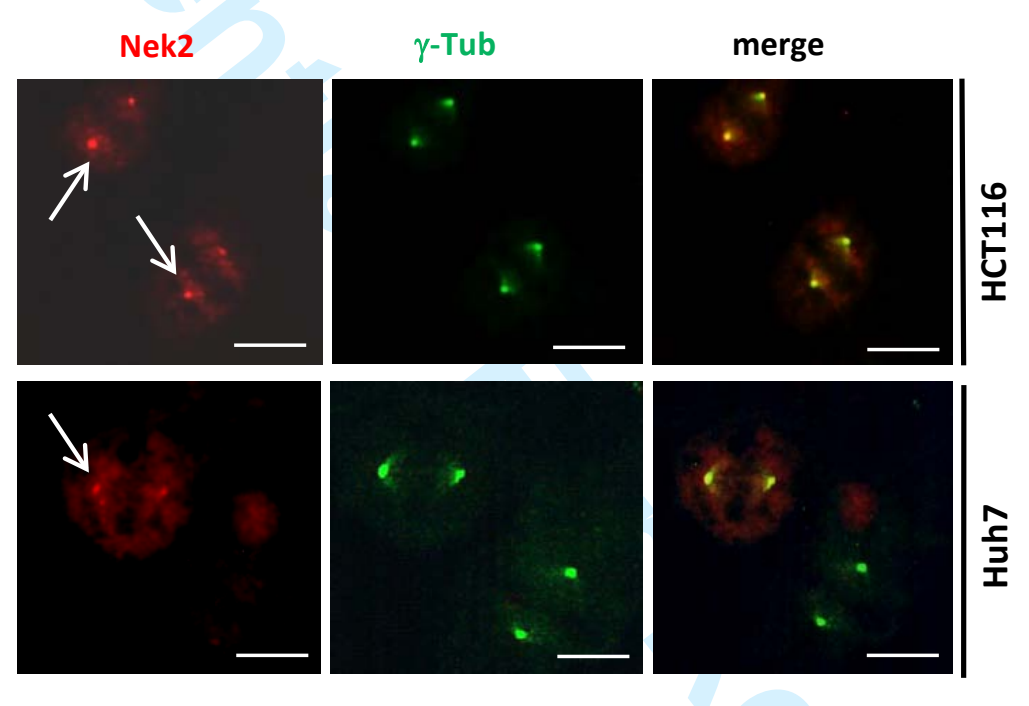

C

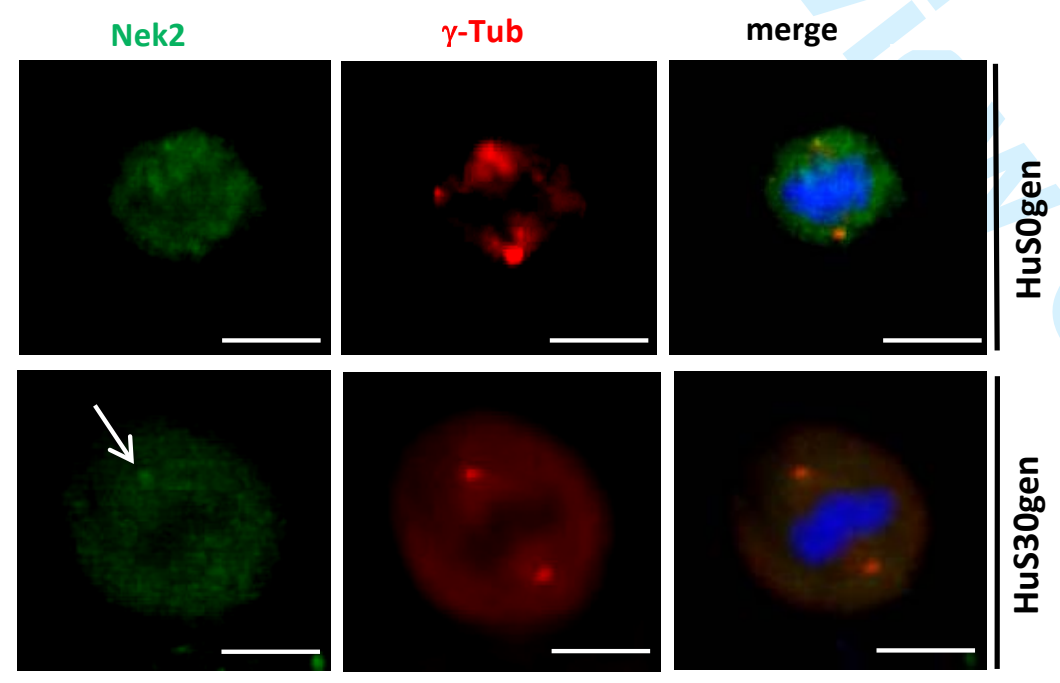

https://mc.manuscriptcentral.com/gut

56

57 